\title{
Heme Oxygenase (HO)-1 Induction Prevents Endoplasmic Reticulum Stress-Mediated Endothelial Cell Death and Impaired Angiogenic Capacity
}

Hatem Maamoun ${ }^{1}$, Matshediso Zachariah ${ }^{1}$, John $\mathrm{H} \mathrm{McVey}^{1}$, Fiona R Green ${ }^{1}$ and Abdelali Agouni ${ }^{2 *}$

${ }^{1}$ University of Surrey, Faculty of Health and Medical Sciences, School of Biosciences \& Medicine, Guildford, GU2 7XH, United Kingdom.

${ }^{2}$ Qatar University, College of Pharmacy, Pharmaceutical Sciences Section, P.O. Box 2713, Doha, Qatar.

\section{"Correspondence:}

Dr. Abdelali Agouni

Pharmaceutical Sciences Section

College of Pharmacy

Qatar University

P.O. Box 2713, Doha, Qatar.

Phone: +97444035610 .

E-mail: aagouni@qu.edu.qa

\section{Classification}

Cardiovascular Pharmacology 


\begin{abstract}
Most of diabetic cardiovascular complications are attributed to endothelial dysfunction and impaired angiogenesis. Endoplasmic Reticulum (ER) and oxidative stresses were shown to play a pivotal role in the development of endothelial dysfunction in diabetes. Hemeoxygenase-1 (HO-1) was shown to protect against oxidative stress in diabetes; however, its role in alleviating ER stress-induced endothelial dysfunction remains not fully elucidated. We aim here to test the protective role of HO-1 against high glucose-mediated ER stress and endothelial dysfunction and understand the underlying mechanisms with special emphasis on oxidative stress, inflammation and cell death.
\end{abstract}

Human Umbilical Vein Endothelial Cells (HUVECs) were grown in either physiological or intermittent high concentrations of glucose for 5 days in the presence or absence of Cobalt (III) Protoporphyrin IX chloride (CoPP, HO-1 inducer) or 4-Phenyl Butyric Acid (PBA, ER stress inhibitor). Using an integrated cellular and molecular approach, we then assessed ER stress and inflammatory responses, in addition to apoptosis and angiogenic capacity in these cells.

Our results show that HO-1 induction prevented high glucose-mediated increase of mRNA and protein expression of key ER stress markers. Cells incubated with high glucose exhibited high levels of oxidative stress, activation of major inflammatory and apoptotic responses

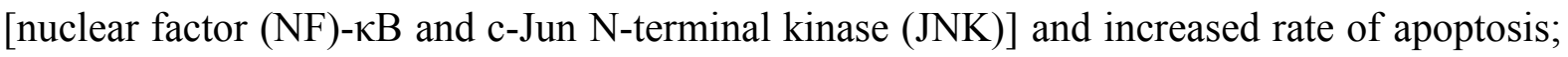
however, cells pre-treated with CoPP or PBA were fully protected. In addition, high glucose enhanced caspases 3 and 7 cleavage and activity and augmented cleaved poly ADP ribose polymerase (PARP) expression whereas HO-1 induction prevented these effects. Finally, HO-1 induction and ER stress inhibition prevented high glucose-induced reduction in NO release and impaired the angiogenic capacity of HUVECs, and enhanced vascular endothelial growth factor (VEGF)-A expression. 
Altogether, we show here the critical role of ER stress-mediated cell death in diabetesinduced endothelial dysfunction and impaired angiogenesis and underscore the role of HO-1 induction as a key therapeutic modulator for ER stress response in ischemic disorders and diabetes. Our results also highlight the complex interplay between ER stress response and oxidative stress.

Key words: Endothelial dysfunction, ER stress, oxidative stress, Heme oxygenase-1

Compounds: Cobalt (III) Protoporphyrin IX chloride (CoPP, PubChem CID:9961203), 4Phenyl Butyric Acid (PBA, PubChem CID:4775), Sn Protoporphyrin IX dichloride (SnPP, PubChem CID: 73755113). 


\section{Introduction}

Endothelial dysfunction generally refers to structural and functional impairment of endothelium including damage at the cellular level. It plays a key role in the pathogenesis of various cardiovascular diseases, such as atherosclerosis, diabetic complications, and hypertension. Heme oxygenase (HO) is one of the cyto-protective proteins that could confer a beneficial effect in vasculature. HO originally functions as a rate-limiting enzyme in heme degradation, yielding carbon monoxide (CO), iron, and biliverdin as the end products. HO-1 and $\mathrm{HO}-2$ are isoforms of $\mathrm{HO}$ in mammals [1]. The HO-2 isoform is constitutively expressed; however, HO-1 is an inducible one. HO-1 expression can be induced by many structurally different molecules and by cellular stress such as heat shock [2]. In particular, the role of hemeoxygenase-1 (HO-1) as a protective enzyme is well known. Its anti-oxidant, antiapoptotic, and anti-inflammatory effects have been extensively studied [2, 3]. The cytoprotective effect of HO-1 may have several distinct underlying mechanisms, including the degradation of heme to the anti-oxidant bilirubin, the co-ordinate induction of ferritin, which chelates the pro-oxidant free iron, and release of carbon monoxide (CO), which exerts significant anti-inflammatory and anti-apoptotic effects [4].

HO-1 can be induced by numerous oxidizing agents and stimuli, including ultraviolet radiation, heavy metals, cytokines and heme/hemoglobin $[2,5]$. It is now well established that HO-1 can provide anti-oxidation and cyto-protection in in vitro and in vivo systems. There is convincing evidence indicating that $\mathrm{HO}-1$ can protect the vasculature against resinamodeling and endothelial dysfunction [6]. Furthermore, HO-1 is currently regarded as a novel therapeutic target in the treatment of vascular disease, and several strategies have been employed to target this enzyme in the vasculature. A variety of pharmacological agents of different chemical structures have been shown to protect against the development of vascular disease in numerous studies in vivo [7, 8]. Moreover, Yang et al. [9] demonstrated the anti- 
oxidant protective role of HO-1 in vitro by reducing endogenous reactive oxygen species (ROS) production in HUVECs when grown in serum from rats exposed to cigarette smoke [9]. Concerning the anti-inflammatory role of HO-1, Chang et al. [10] successfully highlighted this role in HUVECs treated with iodine-based contrast media that was used as an inflammatory model in this study. They showed that HO-1 induction reduced ICAM-1 and adhesion molecules receptor expression [10]. About the anti-apoptotic effects of HO-1, Abraham et al. [11] have previously reported that the overexpression of HO-1 in human dermal microvessel endothelial cells prevented high glucose-mediated slowdown of cell cycle progression and increase in apoptosis [11]. Utilizing Zucker diabetic fat rats (ZDF), it has also been reported that the induction HO-1expression at the early stages of diabetes using cobalt protoporphyrin $(\mathrm{CoPP})$ and stannous chloride $\left(\mathrm{SnCl}_{2}\right)$ imporved blood pressure and reduced endothelial cell sloughing and strongly upregulated anti-apoptotic signals including Bcl-xl and Bcl-2 [12].

Despite the cyto-protective effects of HO-1 that have been well-documented in literature, the role it plays with regards to ER stress response which has been shown in recent years to be involved in the pathophysiology of insulin resistance/diabetes [13] and in the development of endothelial dysfunction [14] is not fully elucidated. The ER is the site of protein secreted synthesis and folding. Upon disruption in protein folding or modification within the ER, a state of stress ensues, resulting in the unfolded protein response (UPR) with the aim to return the ER to its physiological state and enhance cell survival [15-17]. The UPR functions via signalling through three stress-sensing proteins found on the ER membrane: PKR-like eukaryotic initiation factor $2 \alpha$ kinase (PERK), inositol-requiring kinase-1 $\alpha$ (IRE-1 $\alpha$ ), and activating transcription factor (ATF)-6. The ER luminal domains of PERK, IRE-1 $\alpha$, and ATF-6 interact with the ER chaperone Binding immunoglobulin protein (BiP). BiP is responsible for maintaining the effectors of UPR response under inactive form. Under stress 
conditions, accumulation of misfolded or unfolded proteins within ER, BiP dissociates from these sensor proteins, which thereby become active. However, prolonged UPR activation leads to apoptosis, oxidative stress, and inflammation and is referred to as the "ER stress response". Diabetes results in metabolic conditions that increase the demand on the ER for protein and lipid synthesis [15]. Several studies including ours have shown that insulin resistant states such as obesity promote ER stress response in various tissues such as liver, adipose tissue, and the heart $[13,18,19]$.

Activated PERK phosphorylates, and thus activates, eIF (eukaryotic initiation factor)-2 $\alpha$, thereby reducing general protein translation. However, the translation of ATF-4 is selectively permitted. ATF-4 is required for the expression of pro-apoptotic CCAAT/enhancer-binding protein homologous protein $(\mathrm{CHOP})$ transcription factor that plays a key role to ER stressmediated cell dysfunction. CHOP upregulates expression of pro-apoptotic proteins such as Bim, and downregulates expression of anti-apoptotic molecule Bcl-2 [16, 17]. ER stress causes insulin resistance and participates in the low-grade inflammation observed in insulin resistant states $[13,20]$ by mediating cell death and the activation of inflammatory pathways such as nuclear factor- $\kappa \mathrm{B}(\mathrm{NF}-\kappa \mathrm{B})$ and c-Jun N-terminal kinase (JNK) [15]. An association between ER stress and endothelial dysfunction was reported in experimental models of diabetes $[14,21]$; however, the underpinning mechanisms are unclear especially regarding the role of ER-stress mediated inflammation and cell death.

The aim of this study was to show in the context of a high glucose environment, how HO-1 induction would affect ER stress-induced endothelial dysfunction and angiogenic capacity in endothelial cells. As such, we hypothesized that HO-1 would reduce ER stress response activity and hence improve ER stress-mediated inflammation, oxidative stress and apoptosis, which are major molecular contributors to endothelial dysfunction, the initial step in the development of atherosclerosis. 
By using human vascular endothelial cells as a model, we provide evidence that HO-1 induction exerts a protective role against ER stress-mediated endothelial dysfunction and impaired angiogenic capacity caused by high glucose treatment. The effects of HO-1 induction involve several protective mechanisms including the alleviation of oxidative stress in addition to inflammatory and apoptotic responses induced by ER stress activation. We show here, the critical role of ER stress-mediated cell death in diabetes-induced endothelial dysfunction and impaired angiogenic capacity. These results underscore the role of HO-1 induction as a key modulator for ER stress response in metabolic disorders and diabetes. 


\section{Materials \& Methods}

\subsection{Cell culture and treatments}

Human Umbilical Vein Endothelial cells (HUVECs) were obtained from Life Technologies (Paisley, UK) and were routinely cultivated in M200 medium (Gibco, Paisley, UK) supplemented with low serum growth supplement (LSGS; Gibco, Paisley, UK) containing $2 \%$ of foetal bovine serum and were maintained at $37^{\circ} \mathrm{C}$ in a humidified atmosphere with $5 \%$ $\mathrm{CO}_{2}$. Cells were used for experiments up to passage 6 .

To assess the effects of high glucose on endothelial cells, HUVECs were incubated either with culture medium containing a physiological concentration of glucose $(5 \mathrm{mM})$ or cultured in high glucose $(33 \mathrm{mM})$ for 5 days with intermittent recovery periods of 8 hours with medium containing $5 \mathrm{mM}$ of glucose $[16$ hours in high glucose $(33 \mathrm{mM})$ followed by 8 hours in normal glucose $(5 \mathrm{mM})$ for 5 consecutive days]. M200 culture medium was supplemented with D-glucose (SigmaAldrich, Gillingham, UK) to the desired concentration. To factor out the osmotic stress effect which high glucose treatments may have cells, mannitol (SigmaAldrich, Gillingham, UK) was used as an osmotic stress control where a control groups of HUVECs were treated in similar fashion to the high glucose group, with medium supplemented with mannitol $(33 \mathrm{mM})$ with 8 hours recovery periods for 5 days.

To induce HO-1 induction, Cobalt (III) Protoporphyrin IX chloride (CoPP, $10 \mu \mathrm{M}$; SigmaAldrich, Gillingham, UK), a potent HO-1 inducer, was added to HUVECs on the $5^{\text {th }}$ day of glucose treatment 1 hour before the last high glucose treatment and kept for an additional 12 hours before the harvest. A dose- and time-course were established to determine the best CoPP concentration and incubation time to induce $\mathrm{HO}-1$ 's expression in HUVECs. 
To ascertain that effects observed are due to an increase in HO-1's activity, in some experiments cells were treated with Sn-protoporphyrin IX (SnPP) $(20 \mu \mathrm{M}$; SigmaAldrich, Gillingham, UK) which was added to cells 1 hour before CoPP before the last high glucose treatment and then kept for an additional 12 hours before the harvest. SnPP, a metalloporphyrin formed by a chelate of tin with the porphyrin ring, is a potent inhibitor of both HO-1 and HO-2 activities. It induces HO-1 synthesis, but potently inhibits the enzyme's activity by acting as a competitive substrate for heme at the catalytic site [22].

To assess the involvement of ER stress response, some cells were treated with the chemical chaperone, 4-Phenyl Butyric Acid (PBA, 10 mM; SigmaAldrich, Gillingham, UK) to inhibit ER stress [20]. PBA was added to cells 1 hour before the last high glucose treatment and then kept for an additional 12 hours before the harvest.

As a positive control for oxidative stress, some cells were treated with $\mathrm{H}_{2} \mathrm{O}_{2}(100 \mu \mathrm{M}$; SigmaAldrich, Gillingham, UK) for 30 minutes to induce ROS release.

As a positive control for cell death, some cells were stimulated with Staurosporine $(1 \mu \mathrm{M}$; SigmaAldrich, Gillingham, UK) for 12 hours to induce apoptosis.

A positive control for induction of IL-6 synthesis and release, some cells were incubated with lipopolysaccharide (LPS; E. coli serotype O111:B4) $1 \mu \mathrm{g} / \mathrm{ml}$ (Cell Signaling by NEB, Hitchin, UK) at $1 \mu \mathrm{g} / \mathrm{ml}$ for 8 hours prior to assessing IL-6 release in culture medium by ELISA.

\subsection{Total RNA isolation and gene expression analysis}

After incubation of HUVECs with high glucose in the presence or absence of modulators of ER stress response or HO-1 inducer, total RNA was isolated using Qiagen RNeasy mini kit (Qiagen, Manchester, UK) following the manufacturer's protocol. 
Before cDNA synthesis, total RNA was treated with DNAase I to digest any excess genomic DNA. First strand cDNA was synthesized from $1 \mu \mathrm{g}$ of total RNA employing the RevertAid First Strand cDNA Synthesis Kit (ThermoScientific, Loughborough, UK) and an oligo $(\mathrm{dT})_{12-}$ ${ }_{18}$ primer as reverse primer. Then, target genes were amplified by real-time PCR using GoTaq qPCR Master Mix (Promega, Southampton, UK) in Applied Biosystems 7500 Real-Time PCR System (ThermoScientific, Loughborough, UK).

Total cDNA samples with known quantities in $\mathrm{ng}(100 \mathrm{ng} / \mu \mathrm{l}, 10 \mathrm{ng} / \mu \mathrm{l}, 1.0 \mathrm{ng} / \mu \mathrm{l}, 0.1 \mathrm{ng} / \mu \mathrm{l}$ and $0.01 \mathrm{ng} / \mu \mathrm{l})$ were prepared from HUVECs stimulated with thapsigargin. These samples were used to generate standard curves for genes of interest. These standard curves were then used to determine the relative mRNA expression levels in HUVECs.

Primer pairs for target genes were obtained from Primer bank. Human primer sequences used in the study are as follows:

GAPDH_F 5'-CCAGCCGAGCCACATCGCTC -3'

GAPDH_R 5'-ATGAGCCCCAGCCTTCTCCAT -3'

$\beta$-actin_F 5'-CATCACGCCGTCCTATGTCG -3'

$\beta$-actin_R 5'-CGTCAAAGACCGTGTTCTCG -3'

ATF-4_F 5'- GAACGGCTCAAGCAGGAAATC -3'

AFT-4_R 5'- TTCACCATTCGGTCAATCAGAG -3'

BiP_F 5'- GCTGACGATGAAGTTGATGTGG -3'

BiP_R 5'-CATCCGTCCTTGATCCTTCTCTA -3'

CHOP_F 5'-CCCTTCACCTTCTTACAACCTC -3'

CHOP_R 5'- TGCCCAGCTCTAAACTAAAGGA -3' 
IL-6_F 5' - TGATGGCGAAGCGAGTGAAG -3'

IL-6_R 5'- ACTCATCCATACACAGGACCC -3'

VEGF-A_F 5'- GGTGGGCCAAAGGATGAAGAG -3'

VEGF-A_R 5'- CCACAAGCCAAACGACTTCC -3'

\subsection{Western blotting}

After cell treatments, whole-cell lysates were prepared by extraction in radioimmunoprecipitation assay (RIPA) (10 mM Tris-HCl, $\mathrm{pH} 7.4 ; 150 \mathrm{mM} \mathrm{NaCl} ; 0.1 \%$ SDS; $1 \%$ TritonX100; 1\% Sodium deoxycholate; $1 \mathrm{mM} \mathrm{NaF} ; 5 \mathrm{mM}$ EDTA; $1 \mathrm{mM}$ sodium orthovanadate; cocktail of protease inhibitors) as described previously [18, 23]. Proteins of equal amounts (10-20 $\mu \mathrm{g})$ were then separated on $8-12 \%$ SDS-PAGE gels (ThermoScientific, Loughborough, UK). Immunoblots were performed using antibodies against: ATF-4, p-eIF- $\alpha$ (Ser51), caspase 3, cleaved caspase 3, caspase 7, cleaved caspase 7, PARP, cleaved PARP, p-iKK $\alpha / \beta$ (Ser176/180), iKK, p-c-JUN (Ser63), p-Akt (Ser473), Akt, p-eNOS (Ser1177), eNOS (Cell Signaling by NEB, Hitchin, UK), p-p47 $7^{\text {phox }}$ (Ser345), p47 $7^{\text {phox }}$, VEGF-A (SigmaAldrich, Gillingham, UK), BiP, HO-1, $\beta$-actin (Santa Cruz by Insight Biotechnology, Wembley, UK). Proteins were then visualized using fluorescent secondary antibodies and Odyssey CLx infrared imaging system (Li-COR Biosciences, Cambridge, UK) according to manufacturer's guidelines. Signal was then quantified using Image Studio software from LiCOR Biosciences (Cambridge, UK).

\subsection{Assessment of cell death}

Cell apoptosis was assessed by flow cytometry analysis using an Annexin V Apoptosis Detection Kit APC (eBioscience, Altrincham, UK) as described previously [24]. Briefly, after treatments, cells were collected by trypsinization and were re-suspended in incubation buffer containing Annexin V-PE and propidium iodide $(1 \mu \mathrm{g} / \mathrm{ml})$, incubated in the dark at $4{ }^{\circ} \mathrm{C}$ 
for 10 minutes, and then fixed in $1 \%$ formaldehyde. Cells were then re-suspended in incubation buffer and analysed in a BD FACSCanto ${ }^{\mathrm{TM}}$ II (BD Bioscience, Oxford, UK). Cell apoptosis was expressed as the percentage of apoptotic cells expressed as the total number of cells. Some cells were treated with Staurosporine ( $1 \mu \mathrm{M}$; SigmaAldrich, Gillingham, UK) for 3 hours and were used as a positive control for apoptosis while untreated cells were used a negative control.

\subsection{Caspases $3 / 7$ activity}

HUVECs were cultured in physiological concentration of glucose $(5 \mathrm{mM})$ or in intermittent high glucose $(5 / 33 \mathrm{mM})$ for 5 days in the absence or presence of PBA or CoPP. As positive control, some cells were treated with Staurosporine $(1 \mathrm{mM})$ for 3 hours while untreated cells were used a negative control. Then, caspases $3 / 7$ activity was measured using Caspase-Glo ${ }^{\circledR}$ 3/7 Assay (Promega, Southampton, UK) according to the manufacturer's guidelines. Caspase-Glo® 3/7 reagent contains a luminogenic substrate (Aminoluciferin) attached to tetra-peptide sequence Z-DEVD, which is specific for protease cleavage by caspases 3 and 7 . Caspases 3 and 7 cleave Z-DEVD releasing Aminoluciferin which is a luminescent substrate for the luciferase reaction. The light output from the luciferase reaction is directly proportional to the activity of the enzymes. The activity of caspases 3 and 7 was determined using a LUMIstar Omega luminometer (BMG LABTECH, Aylesbury, UK).

\subsection{ELISA analysis of IL-6 production}

HUVECs were incubated in physiological concentration of glucose $(5 \mathrm{mM})$ or in intermittent high glucose $(5 / 33 \mathrm{mM})$ for 5 days in the presence or absence of CoPP $(10 \mu \mathrm{M})$ and/or SnPP $(20 \mu \mathrm{M})$. A positive control was generated by treating some cells with lipopolysaccharide (LPS; Cell Signaling by NEB, Hitchin, UK) at $1 \mu \mathrm{g} / \mathrm{mL}$ for 30 minutes. Supernatants were collected and centrifuged at $10,000 \times \mathrm{g}$ for 10 minutes to rem ove dead cells and any debris 
and then assayed for IL-6 using an ELISA kit (ThermoScientific, Loughborough, UK) according to the manufacturer's instructions.

\subsection{Nitrite and nitrate measurement by Griess assay}

The production of NO was also quantified by measuring the released NO metabolites (nitrite and nitrates) with Griess reagent (Life Technologies, Loughborough, UK). The Griess assay was used to measure the concentration of nitrite/nitrate that are stable by-products of NO degradation as an indirect way of estimating production of NO in HUVECs. Culture medium samples were collected from control and high glucose-treated cells in the presence or absence of PBA or CoPP. Culture medium samples were then centrifuged to remove dead cells and cell debris. The medium was subsequently processed to determine nitrate and nitrite concentrations according to the manufacturer's protocol [25].

\subsection{ROS measurement by dihydroethidine (DHE) staining}

HUVECs were cultured in physiological concentration of glucose $(5 \mathrm{mM})$ or in intermittent high glucose $(5 / 33 \mathrm{mM})$ for 5 days in the absence or presence of PBA or CoPP. As a positive control, $\mathrm{H}_{2} \mathrm{O}_{2}(100 \mu \mathrm{M})$ was added to some cells for 30 minutes to induce ROS release. Osmotic stress was controlled by growing cells in medium where glucose was replaced by mannitol (33 mM). Following this, cells were washed three times with phosphate buffer salt (PBS) solution, and then incubated with the oxidative fluorescent dye DHE $(10 \mu \mathrm{M}$; SigmaAldrich, Gillingham, UK) for 30 minutes. Cells were then harvested, washed three times with PBS and then fluorescence at $620 \mathrm{~nm}$ was determined and the number of DHEpositive cells was calculated using a FACSCanto ${ }^{\mathrm{TM}}$ II flow cytometry (BD Bioscience, Oxford, UK). Negative and positive cells were then plotted using the positive control and DHE negative cells (untreated cells). Cells shifting to the right channel were considered as positive for DHE $[25,26]$. 


\subsection{In vitro tube-like structure formation assay on Matrigel® matrix}

HUVECs were incubated in physiological concentration of glucose $(5 \mathrm{mM})$ or in intermittent high glucose $(33 \mathrm{mM})$ for 5 days in the presence or absence of PBA or CoPP. Then, they were seeded $\left(10^{5}\right.$ cells per well) on 24 -well culture plates coated with a basement membrane preparation extracted from Engelbreth-Holm-Swarm murine sarcoma (Matrigel ${ }^{\circledR}$; BD Bioscience, Oxford, UK) [27]. Briefly, $250 \mu \mathrm{L}$ of Matrigel ${ }^{\circledR}$ substrate diluted with serumfree medium (1:1 dilution) was added into each well of 24-well plates and allowed to solidify for 1 hour at $37^{\circ} \mathrm{C}$. Then, cells were incubated with M200 medium supplemented with LSGS and allowed to adhere for 1 hour. Untreated cells cultured in M200 medium without LSGS were used as the negative control, while those cultured in M200 medium supplemented with the LSGS that has $2 \% \mathrm{FBS}+3 \mathrm{ng} / \mathrm{mL} \beta$-FGF were used as positive control. HUVECs were incubated for further 24 hours and tube-like structures formation was examined using an inverted phase contrast microscope. Then, using ImageJ software, the length of the tubes formed was counted in 5 different blind fields, averaged then compared across the different samples.

\subsection{Assessment of HO-1 activity (Bilirubin assay)}

Bilirubin colorimetric assay kit (Cell Biolabs Inc., San Diego, CA, USA) was used to measure the total and direct bilirubin levels in HUVECs' lysates following the manufacturer's protocol. Briefly, HUVECs were cultured in normal glucose $(5 \mathrm{mM})$ or exposed intermittently to high glucose $(5 / 33 \mathrm{mM})$ for 5 days in the presence or absence of CoPP $(10 \mu \mathrm{M})$ or SnPP $(20 \mu \mathrm{M})$. Then, cells were lysed with RIPA lysis buffer and centrifuged at $10,000 \mathrm{x} g$ for 10 minutes at $4^{\circ} \mathrm{C}$. Bilirubin standards were prepared in 1:2 serial dilutions. Samples and standards were then loaded in duplicates in a 96-well microplate. Accelerant (proprietary reagent) to each well. Diazo reagent was then loaded to samples and standards. Reagent A (Sodium hydroxide) was then added to all wells and the 
plate left for incubation for 1 hour at room temperature protected from light. After incubation is over, assay reagent B was added and absorbance at $540 \mathrm{~nm}$ was measured. The net OD for each sample was calculated by subtracting OD from negative control wells from each sample well, which was then plotted against standard curve to determine bilirubin levels (mg/dL).

\subsection{Cell viability assay}

This viability assay is based on the capacibolity of metabolically active alive cells to covert the yellow tetrazolium salt 3-(4,5-dimethylthiazol-2-yl)-2,5-diphenyltetrazolium bromide (MTT) to purple formazan crystals. This allows a quantitative evaluation of viable cells. HUVECs were seeded into 96-well plates at the density of 20,000 cells per mL and then grown for 24 hours. Following this, cells were incubated with varying concentrations of CoPP $(10,25,50,75$ and $100 \mu \mathrm{M})$ for either 12 or 24 hours. After incubation, we added MTT (SigmaAldrich, Gillingham, UK) at final concentration of $0.5 \mathrm{mg} / \mathrm{mL}$ to each one of the well and incubated cells for further 4 hours at $37^{\circ} \mathrm{C}$. Then, culture medium was removed and $100 \mu \mathrm{L}$ Dimethyl sulfoxide (SigmaAldrich, Gillingham, UK) were added to each well to solubilise the formazan crystals and incubated for further 30 minutes at $37{ }^{\circ} \mathrm{C}$. Absorbance was then measured at $570 \mathrm{~nm}$ using a multi-plate reader (Molecular Devices, Wokingham, UK). The intensity of colour in control wells was used to indicate $100 \%$ of viability.

\subsection{Data analysis}

Results are expressed as mean $\pm \mathrm{SEM}$, and $n$ represents the number of mice or biological replicates. Statistical analyses were performed using ANOVA (2-way or 1-way, as appropriate) followed by Tukey's or Bonferroni is multiple comparison post-hoc tests. $P<$ 0.05 was considered to be statistically significant. 


\section{Results}

\subsection{Validation of the conditions of induction of HO-1 by CoPP in HUVECs}

To validate the optimal conditions for use of CoPP to induce HO-1 in HUVECs and to ensure that the increase in protein levels is indeed reflecting an increase in activity, we conducted a series of experiments. We first constructed a dose- and time-course response to varying concentrations of CoPP to determine the optimal concentration and time for the induction of HO-1 in HUVECs without causing any non-specific toxicity to the cells. Once determined, we evaluated the activity of HO-1 in response to CoPP treatment.

As shown in Figure 1A, the incubation of cells with CoPP at the concentrations of 10, 25, 50, 75 and $100 \mu \mathrm{M}$ all caused an increase in protein expression of HO-1 after 12 and 24 hours; however, the increase was higher at 12 hours. Furthermore, as shown in Figure 1B, the incubation of HUVECs with these different concentrations CoPP for 12 and 24 hours caused a significant decrease in cell viability at 24 hours in all concentrations; however, at 12 hours, none of these concentrations showed a significant decrease in cell viability.

Altogether, we decided for this project to induce HO-1 expression by treating cells with CoPP at the concentration of $10 \mu \mathrm{M}$ for 12 hours.

To ascertain that HO-1 expression reflects an increase in its activity, we have assessed the enzyme's activity by measuring the release of heme degradation by-product bilirubin in cells treated exposed or not to high glucose in the presence or absence of CoPP, a HO-1 inducer, and/or SnPP, a HO inhibitor. As shown in Figure 1C, high glucose did not affect bilirubin levels while the induction of HO-1 by CoPP $(10 \mu \mathrm{M}, 12$ hours $)$ caused an increase of its release indicating an increase in HO-1 activity in HUVECs. The pre-treatment of cells with SnPP partially, but significantly, prevented the increase in bilirubin caused by CoPP. These 
data indicate that in our hands the induction of HO-1 by CoPP reflects an increase of the enzymes activity.

\subsection{HO-1 induction prevents the induction of ER stress response in HUVECs caused by high glucose treatment.}

As expected, intermittent high glucose $(33 \mathrm{mM})$ treatment of HUVECs for 5 days induced an activation of ER stress response. As shown in Figures (2A, 2B and 2C), high glucose caused an increase in mRNA expression of $B i P, C H O P$ and $A T F-4$ to levels comparable to positive control thapsigargin. The pre-treatment of cells with PBA to inhibit ER stress prevented the increase in mRNA expression of these key ER stress markers (Figures 2A, 2B and 2C). At the protein level, similarly to thapsigargin treatment, the incubation of HUVECs with intermittent high glucose enhanced the phosphorylation of e-IF2- $\alpha$ and increased protein expression of both BiP (Figure 2D and 2E) and ATF-4 (Figure 2F). Although mannitol caused a moderate increase in the phosphorylation of e-IF2- $\alpha$, it did not affect the activation of other key ER stress response markers excluding thus any contribution of osmotic stress in high glucose-mediated ER stress response. HO-1 induction by CoPP prevented the activation of ER stress response in HUVECs as evidenced by the normalisation of e-IF2- $\alpha$ phosphorylation and reduction in $\mathrm{BiP}$ and $\mathrm{ATF}-4$ protein expression (Figures 2D, 2E and 2F) indicating a protective role for HO-1 against high glucose-induced ER stress in HUVECs.

\subsection{HO-1 induction prevents oxidative stress caused by high glucose treatment in HUVECs.}

The incubation of HUVECs with intermittent high glucose treatment for 5 days caused a significant increase in ROS production compared to negative control (Figure 3A). HO-1 induction by CoPP significantly reduced ROS production to control levels (Figure 3A) 
indicating the protective role that HO-1 has against high glucose-induced oxidative stress. Mannitol failed to show any increase in ROS production indicating that these effects shown with high glucose treatment are due to metabolic stress and not osmotic stress. PBA treatment also significantly reduced ROS production caused by high glucose although not to the same extent as CoPP (Figure 3A). These data indicate a considerable degree of ER stress involvement in high glucose-mediated increase in ROS production.

NADPH oxidase system is an important source of ROS production in the vasculature particularly in endothelial cells [28]. As shown in Figure 3B, when HUVECs were subjected to high glucose (33 $\mathrm{mM}$ for 5 days with intermittent recovery periods), there was a significant increase in the phosphorylation of $\mathrm{p} 47^{\text {phox }}$ regulatory subunit when compared to control condition; whereas, co-treatment of cells with CoPP has resulted in a significant reversal of such effect. The phosphorylation of regulatory subunit $\mathrm{p} 47^{\text {phox }}$ is believed to be a prerequisite for the activation of NADPH oxidase complex [29]. The induction of ER stress response by thapsigargin also enhanced the phosphorylation of $\mathrm{p} 47^{\mathrm{phox}}$ and this effect was prevented by pre-incubation of cells with PBA, again indicating the involvement of ER stress in this process.

\subsection{HO-1 induction prevents ER stress-mediated activation of pro-inflammatory signalling pathways caused by high glucose.}

Intermittent treatment of HUVECs with high glucose for 5 days significantly increased phosphorylation of inflammatory markers iKK $\alpha / \beta$ (Figure 4A) and c-JUN (Figure 4B) compared to control. This effect was also noticeable with thapsigargin treatment and reversed by PBA indicating involvement of ER stress in this process. When adding PBA to the cells incubated with high glucose, similarly to thapsigargin this has prevented the increase in phosphorylation of both iKK $\alpha / \beta$ and c-JUN (Figure $4 \mathbf{A}$ and $4 \mathbf{B}$ ) indicating that high glucose-induced inflammatory response is related to ER stress response induction. Moreover, 
HO-1 induction by CoPP significantly prevented the increase in phosphorylation of both iKK $\alpha / \beta$ and c-JUN (Figure 4A and 4B) indicating a cross-talk between ROS and ER stress response in this process. To further emphasize the involvement of oxidative stress in inflammatory response, we were able to observe significant increase in the phosphorylation of both inflammatory markers iKK $\alpha / \beta$ and c-JUN (Figure 4A and 4B) when HUVECs were treated with $\mathrm{H}_{2} \mathrm{O}_{2}(100 \mu \mathrm{M})$ for 30 minutes. HO-1 induction prevented the effects of $\mathrm{H}_{2} \mathrm{O}_{2}$ on phosphorylation of $\mathrm{iKK} \alpha / \beta$ and c-JUN. To exclude the involvement of osmotic stress in this inflammatory response, cells were treated with mannitol which did affect the neither the expression nor the activation of inflammatory molecules investigated (Figure 4A and 4B).

In order to ascertain the protective role $\mathrm{HO}-1$ has against high glucose-induced inflammatory response in HUVECs, we assessed the production of a key pro-inflammatory cytokine IL-6. As shown above in Figure 4C, similarly to thapsigargin, IL-6 mRNA levels have significantly increased after treatment of cells with intermittent high glucose compared to controls. This increase was significantly reversed back to control levels with PBA treatment indicating ER stress involvement in this process (Figure 4C). HO-1 induction by CoPP reversed the increase in IL- 6 mRNA expression both in high glucose and thapsigargin-treated HUVECs to bring them back to control levels (Figure 4C). This suggests that the antioxidant activity of HO-1 plays a protective role against high glucose-induced inflammation, at least partly, through the alleviation of ER stress response. Furthermore, ELISA analysis of IL-6 protein release in culture medium collected from HUVECs showed a significant elevation in IL-6 levels in high glucose culture medium compared to control (Figure 4D). However, culture medium harvested from HUVECs treated with high glucose in the presence of CoPP showed similar levels of IL-6 expression to negative control clearly indicating a protective effect of HO-1 induction against high glucose-mediated inflammation. When SnPP $(20 \mu \mathrm{M})$, 
a heme analogue inhibitor of $\mathrm{HO}$, was added 1 hour before CoPP treatment, this has negated CoPP effects on high glucose-mediated increase of IL-6 release (Figure 4D).

\subsection{HO-1 induction prevents ER stress-mediated apoptosis caused by high glucose treatment in HUVECs: Critical role for caspases 3 and 7.}

Analysis of apoptosis by flow cytometry showed that amongst HUVECs cultured in intermittent high glucose conditions, the percentage of apoptotic cells significantly increased to $45 \%$ compared to $7 \%$ in HUVECs cultured in medium with physiological glucose concentration (Figure 5). However, the percentage of apoptotic cells amongst HUVECs cultured in intermittent high glucose media was significantly reduced to $15 \%$ after $\mathrm{HO}-1$ induction with CoPP treatment (Figure 5). To further emphasize the anti-apoptotic effect of HO-1 induction, SnPP, a HO inhibitor $(20 \mu \mathrm{M})$, was added 1 hour prior to adding CoPP to HUVECs incubated with high glucose. SnPP reversed the protective effects of HO-1 with a significant increase in percentage of apoptotic cells to $40 \%$ (Figure 5), thus providing further evidence on the protective effect of HO-1 against apoptosis in high glucose milieu and indicating the key role of ER stress in this process.

To further understand the mechanisms underlying the protective role of HO-1 against high glucose-induced apoptosis, we assessed the enzymatic activity of caspases 3/7. As shown in Figure 6A, HUVECs cultured in intermittent high glucose $(33 \mathrm{mM})$ conditions showed a significant increase in caspases $3 / 7$ activity compared to negative control. This effect was negated by CoPP treatment bringing caspases $3 / 7$ activity levels to those of the negative control.

Furthermore, we determined by western blot analysis the expression of active (cleaved) caspases 3/7 and PARP (a caspase-3 substrate). As shown in Figure 6B, HUVECs incubated with high glucose medium (33 mM) exhibited cleavage of caspases 3/7 and PARP indicating 
the activation of these pro-apoptotic sub-pathways. Similar observations were also noticeable with thapsigargin treatment. When adding PBA to cells incubated with high glucose, has prevented the cleavage of caspases $3 / 7$ and PARP indicating that high glucose-induced apoptotic response involves ER stress response induction (Figure 6B).

Furthermore, we analysed the cleavage of these three pro-apoptotic effectors (PARP and caspases 3/7) following the treatment of HUVECs with $\mathrm{H}_{2} \mathrm{O}_{2}(100 \mu \mathrm{M})$ for 30 minute. $\mathrm{H}_{2} \mathrm{O}_{2}$ caused the cleavage of PARP and caspases $3 / 7$ and this effect was abolished by HO-1 induction with CoPP treatment. Cells incubated with high glucose and where HO-1 was induced by CoPP showed no cleavage of these proteins indicating the protective role HO-1 plays against oxidative stress-mediated apoptosis (Figure 6B). When cells were treated with mannitol, no cleavage of caspases $3 / 7$ or PARP was observed which excludes the involvement of osmotic stress in this apoptotic response.

Altogether, our data show that HO-1 induction was able to prevent apoptosis triggered by oxidative stress and ER stress response following exposure of endothelial cells to high glucose. These effects were related to the inhibition of NF-kB and JNK pro-apoptotic and inflammatory pathways, alleviation of ER stress response particularly the reduction of CHOP expression and to preventing the cleavage of caspases 3/7 and PARP, which play a key central role in the final steps of different apoptosis cascades.

\subsection{Alleviation of high glucose-induced ER stress by HO-1 induction improves endothelial function and angiogenic capacity in HUVECs.}

As shown in Figure 7A, high glucose treatment decreased NO production in HUVECs while the induction of HO-1 prevented this effect increasing thus NO bioavailability considering the concomitant decrease in ROS observed previously (Figure 3). Furthermore, western blot analysis of key components of NO pathway, showed that HO-1 induction by CoPP strongly 
enhanced the phosphorylation of eNOS on its key activatory site (Ser 1177) and the phosphorylation of Akt (Ser 473) (Figure 7B and 7C) indicating the activation of Akt/eNOS that is involved in the positive regulation of NO release. As presented in Figure 7D, HO-1 expression was successfully induced by CoPP treatment $(10 \mu \mathrm{M})$ while high glucose (33 $\mathrm{mM}$ ) did not affect the protein's expression.

As depicted in Figure 8A, HUVECs treated intermittently with high glucose for 5 days showed significant reduction in tube-like structure formation compared to positive control. This effect was reversed by PBA treatment with significant increase in tube-like structure formation compared to high glucose alone indicating the involvement of ER stress in this process (Figure 8A). Moreover, similar protective effect was noticeable with HO-1 induction by CoPP indicating its protective pro-angiogenic role and antagonising the effects of high glucose-induced ER stress. This pro-angiogenic effect of HO-1 induction was further supported by the observation that CoPP treatment also caused an increase in mRNA (Figure 8B) and protein (Figure 8C) expression of the key pro-angiogenic factor VEGF-A in endothelial cells.

Altogether, these findings provide evidence that the protective role of $\mathrm{HO}-1$ induction by CoPP against impaired angiogenesis caused by high glucose in HUVECs is carried out by activating the pro-angiogenic VEGF-A - p-eNOS/P-AKT-NO axis and attenuation ER stress response. 


\section{Discussion}

In this study, we provide evidence that HO-1 induction exerts a protective role against ER stress-mediated endothelial dysfunction and impaired angiogenic capacity caused by high glucose treatment. The effects of HO-1 induction involve several protective mechanisms including the alleviation of oxidative stress in addition to inflammatory and apoptotic responses induced by ER stress activation.

High glucose-mediated ER stress response is believed to play a key role in different aspects of endothelial dysfunction both in humans and in animal models of diabetes. High glucose induces ER stress response and ROS production in a close interplay, which ultimately may contribute to the pathogenesis of micro- and macro-vascular complications, associated with diabetes [31, 32]. Recently, mice injected with streptozotocin to cause diabetes exhibited enhanced ER stress response in the heart and small mesenteric arteries. Treatment of these mice with a chemical chaperone (Tauroursodeoxycholic acid, TUDCA) improved endothelium-dependent and -independent relaxation. We report here that exposure of HUVECs to high glucose caused an increase in both mRNA and protein expression of key ER stress markers (phospho-eIF2- $\alpha, \mathrm{BiP}, \mathrm{CHOP}$ and ATF-4) indicating that the upstream ER stress arm PERK/eIF2- $\alpha$ is activated under these conditions. PERK is the major effector controlling the attenuation of protein translation when ER stress ensues through the activation of the elongation factor, eIF2- $\alpha$. Activation of eIF2 $\alpha$ allows for selective translation of ATF-4 that regulates the expression of the pro-apoptotic factor CHOP. We also report that similarly to chemical chaperone PBA, the induction of HO-1 by CoPP, reversed the effects of high glucose on ER stress indicating an important cross talk between ROS and ER stress in these conditions. Consistent with our observations, Kim et al. [33] have reported that that induction of HO-1 through the exposure of HUVECs to $\mathrm{CO}$ or CoPP prevented thapsigargin-induced expression of CHOP [33]. 
Mannitol, however, caused a moderate increase in the expression phospho-eIF2- $\alpha$ without affecting the expression of other ER stress markers. This is possibly because mannitol may have enhanced a transient need for more protein synthesis and hence caused a transient activation of UPR response.

During ER stress, several mechanisms can lead to increased ROS production including the ER itself, which becomes an important source of ROS due to the inhibition of protein translation by the PERK/eIF2- $\alpha /$ ATF-4 pathway, in addition to the activation of NADPH oxidase complex [34]. As expected, we found here that high glucose-mediated ER stress increased ROS production in HUVECs that was blunted by HO-1 induction and PBA indicating a strong interplay between ER stress and ROS in high glucose conditions. NADPH oxidase is a major source of ROS production in the vasculature [28]. Among other important regulatory mechanisms, the phosphorylation of $\mathrm{p} 47^{\text {phox }}$ regulatory subunit was shown to be a prerequisite for the activation of NADPH oxidase [29]. We observed here that the phosphorylation of $\mathrm{p} 47^{\text {phox }}$ at Ser345 was enhanced in cells exposed intermittently to high glucose suggesting the activation of NADPH oxidase complex. The induction of HO-1 by CoPP or the alleviation of ER stress response by PBA reduced the phosphorylation of $\mathrm{p} 47^{\text {phox }}$ indicating the involvement of ER stress in this process. To the best of our knowledge, this is the first time that HO-1 induction or ER stress inhibition was reported to prevent high glucose-mediated serine phosphorylation of $\mathrm{p} 47^{\text {phox }}$ in endothelial cells. The phosphorylation of $\mathrm{p} 47^{\text {phox }}$ implies a central role for ER stress in the activation of serine/threonine kinase(s) that can phosphorylate $\mathrm{p} 47^{\text {phox }}$. High glucose-mediated ER stress can increase intracellular $\mathrm{Ca}^{2+}$ release from the ER, which, in turn, can cause the activation of protein kinase $\mathrm{C}$ [35] which was recently reported to promote the serine phosphorylation of $p 47^{\text {phox }}[36]$. However, the exact role of this phosphorylation and the activation of NADPH oxidase warrants further 
investigation to establish a clear mechanistic link between HO-1, ER stress and activation of NADPH oxidase.

Much evidence links ER stress with major inflammatory signalling hubs. For instance, PERK/ eIF2- $\alpha /$ ATF-4 arm of ER stress triggers the activation of JNK which is involved in the positive regulation of some inflammatory genes and apoptosis [34]. In our hands, high glucose-mediated ER stress was associated with the activation of JNK and NF- $\kappa$ B pathways and caused a significant increase in the production of IL-6 inflammatory cytokine. All these effects were counteracted by HO-1 induction and the inhibition of ER stress indicating the involvement of ROS either upstream and/or downstream of ER stress in this process. Inflammatory response has been mechanistically linked to oxidative stress [37, 38]. Consistent with our findings, previous studies indicated that hyperglycaemia triggers the generation of free radicals which activate NF- $\kappa \mathrm{B}$ that is known to induce the up-regulation of other pro-inflammatory cytokines including IL-6, which can in turn activate ER stress response by a vicious cycle and contribute to the maintenance of the inflammatory state during ER stress [39].

We observed here that HO-1 induction alleviated the effects of high glucose in activating several pro-apoptotic pathways, in which ER stress is central, including the activation of JNK and PERK/eIF2- $\alpha /$ ATF-4/CHOP pathways. Several mechanisms controlling cell death following unresolved ER stress have been reported most of which have been prevented by HO-1 induction in the present study [40]. In prolonged ER stress, IRE-1 $\alpha$ sensor can cause the phosphorylation and activation of JNK, which can ultimately induce apoptosis [16]. When ER stress becomes severe, the PERK/eIF2 $\alpha /$ ATF-4 pathway also activates the transcription factor $\mathrm{CHOP}$, which upregulates expression of pro-apoptotic proteins such as Bim, and downregulates the expression of anti-apoptotic molecule Bcl-2 [16, 17]. 
Consistent with other studies, we report here that the activation of JNK and PERK/eIF2$\alpha / \mathrm{ATF}-4 / \mathrm{CHOP}$ axis caused by high glucose was associated with a high apoptosis rate in HUVECs [41]. HO-1 induction or inhibition of ER stress prevented the activation of these pro-apoptotic signals, which was translated into a reduction of apoptosis. These findings further highlight the important anti-apoptotic role of HO-1. Endogenous HO-1 induction was observed in HUVECs exposed to high glucose levels up to $10 \mathrm{mM}$; however, higher concentrations of glucose $(>20 \mathrm{mM})$ did cause a significant downregulation of $\mathrm{HO}-1$ expression which was linked to an increase in apoptosis rate [41]. Another study by Abraham et al. [11] also found that incubation of endothelial cells with high glucose $(33 \mathrm{mM})$ for 7 days caused a decrease in $\mathrm{HO}$ activity and protein expression [11]. This suggests that $\mathrm{HO}-1$ levels are critical to overcome the pro-apoptotic effects of high glucose. In our study and consistent with these previous studies, we found that high glucose $(33 \mathrm{mM})$ did not induce HO-1 expression or activity. We observed that these high concentrations of glucose $(33 \mathrm{mM})$ failed to increase apoptosis in HUVECs in the presence of HO-1 inducer, CoPP, indicating a therapeutic potential for HO-1 induction against high glucose-mediated endothelial apoptosis. These pro-survival effects of HO-1 are directly linked to the enzyme's activity because the treatment of HUVECs with SnPP, a potent HO inhibitor, reversed the beneficial effects of HO-1 induction on cell survival.

An important feature of apoptosis is the activation of caspases where the Bcl-2 family plays a crucial role in regulating their engagement under ER stress [42]. In our study, we observed that HO-1 induction protected HUVECs against high glucose-induced activation of caspases 3 and 7. Consistent with our results, it was found that under ER stress conditions, through the action of JNK-dependent signalling, caspase-3 cleavage and activity is enhanced leading to high levels of calcium in the mitochondria [43]. In our study, the activation of caspase-3 
effector is consistent with the activation by high glucose of PERK/eIF2- $\alpha /$ ATF-4/CHOP pathway.

Perturbations in angiogenesis are tightly linked to endothelial dysfunction and contribute to ischemic complications of diabetes. HO-1 was found to possess potent pro-angiogenic properties. The overexpression of $\mathrm{HO}-1$ in endothelial cells was reported to enhance cell proliferation and tube formation capacity [44]. We report here for the first time that HO-1 induction and inhibition of ER stress in HUVECs prevented the impairment of capillary-like structure formation on Matrigel assay caused by intermittent high glucose treatment indicating the involvement of ER stress in this process and the therapeutic potential of HO-1 induction against high glucose-mediated ischemia. In line with our findings, a number of studies implicated ER stress in endothelial dysfunction and impaired angiogenic capacity. For instance, the chemical chaperone TUDCA was shown to protect against impaired ischemiainduced neovascularization following hind-limb ischemia after femoral artery ligation in $d b / d b$ diabetic mice [45]. Endothelial dysfunction plays a key role in diabetic microvascular dysfunction because endothelial NO is a crucial regulator of angiogenic sprouting [46]. We report here that HO-1 induction and ER stress inhibition improved NO production and enhanced the activation of Akt/eNOS pathway in HUVECs exposed to high glucose, which eventually contributes to restoring the capacity of cells to form tube-like structures. The concomitant reduction of ROS production in cells treated with CoPP or PBA also contributes to the improved NO bioavailability. ER stress can affect NO bioavailability through several ways and hence impair angiogenic capacity of endothelial cells. Accumulation of CHOP was shown to inhibit eNOS synthesis through a direct interaction with the enzyme's promoter while CHOP-deficient mice exhibited an improved postnatal ischemia-induced neovascularization [47]. ER stress can also affect endothelial NO bioavailability by causing endothelial insulin resistance which may directly and negatively affect endothelial function. 
Insulin by binding to its receptor, expressed by endothelial cells, can stimulate the release of NO by endothelial cells [48], and, thus, endothelial insulin resistance can impair insulinmediated NO release [49]. Ozcan et al. reported that the activation of ER stress enhanced NF$\kappa \mathrm{B}$ and JNK pathways, which then phosphorylated insulin receptor substrate (IRS)-1 on serine residues and hence blunted insulin signalling response [13]. In our study, we found that high glucose-mediated ER stress was closely associated with the activation of NF- $\kappa \mathrm{B}$ and JNK pathways, which may in turn contribute to the impairment of endothelial insulin response.

Although, the role that heme degradation by-products play in the cyto-protective effects of HO-1 in our system was not specifically studied, some evidence in the literature indicate that the action of both bilirubin and CO may alleviate ER stress response; however, their exact role needs to be further delineated in future studies. Biliverdin and bilirubin are both potent antioxidants through a direct ROS scavenging mechanism and were found to prevent oxidative stress-mediated cell death [50]. Recently, bilirubin was shown to enhance insulin signalling response in a diet-induced obesity mouse model an in $d b / d b$ diabetic mice through the suppression of ER stress response [51]. Bilirubin levels in humans were found to be associated with reduced risk for coronary heart disease $[50,52]$. $\mathrm{CO}$, the other degradation by-product, was found to prevent apoptosis in endothelial cells by activating p38 MAP kinase. $\mathrm{CO}$ was also shown to improve insulin response by increasing intracellular calcium [50]. Recently, it was reported that CO- releasing agent, CORM-2, improved ER stress response and blocked the ER stress-mediated impairment in leptin signalling in cells treated with ER stress inducers thapsigargin and tunicamycin [53].

Altogether, we demonstrate in the present study the pivotal role of ER stress-mediated cell death in high glucose-induced endothelial dysfunction and impaired angiogenic capacity and 
underscore the potential role of HO-1 induction as a key therapeutic modulator for ER stress response in ischemic disorders and diabetes. 


\section{Conflict of interest}

None to declare.

\section{Acknowledgments}

This work was supported with grants to Dr Abdelali Agouni from the Royal Society, the Physiological Society, and Qatar University (grant QUUG-CPH-CPH-15/16-6). Mr Maamoun and Ms. Zachariah are supported by doctoral scholarships from Egyptian cultural bureau and Government of Botswana, respectively.

The graphical abstract was produced by adapting freely available tools from Servier Medical Art, which are licensed under a Creative Commons Attribution 3.0 Unported License https://creativecommons.org/licenses/by/3.0/ 


\section{References:}

[1] H. Yang, S.E. Lee, G.D. Kim, H.R. Park, Y.S. Park, Hemeoxygenase-1 mediates an adaptive response to spermidine-induced cell death in human endothelial cells, Oxid Med Cell Longev 2013 (2013) 238734.

[2] N.G. Abraham, A. Kappas, Pharmacological and clinical aspects of heme oxygenase, Pharmacol Rev 60(1) (2008) 79-127.

[3] M. Zheng, S.K. Kim, Y. Joe, S.H. Back, H.R. Cho, H.P. Kim, L.J. Ignarro, H.T. Chung, Sensing endoplasmic reticulum stress by protein kinase RNA-like endoplasmic reticulum kinase promotes adaptive mitochondrial DNA biogenesis and cell survival via heme oxygenase-1/carbon monoxide activity, FASEB J 26(6) (2012) 2558-68.

[4] W. Durante, Targeting heme oxygenase-1 in vascular disease, Curr Drug Targets 11(12) (2010) 1504-16.

[5] M.D. Maines, The heme oxygenase system: a regulator of second messenger gases, Annu Rev Pharmacol Toxicol 37 (1997) 517-54.

[6] W. Durante, Heme oxygenase-1 in growth control and its clinical application to vascular disease, J Cell Physiol 195(3) (2003) 373-82.

[7] J.M. Hyvelin, B. Maurel, R. Uzbekov, R. Motterlini, P. Lermusiaux, Hemin prevents instent stenosis in rat and rabbit models by inducing heme-oxygenase-1, J Vasc Surg 51(2) (2010) 417-28.

[8] T. Li, H. Tian, Y. Zhao, F. An, L. Zhang, J. Zhang, J. Peng, Y. Zhang, Y. Guo, Heme oxygenase-1 inhibits progression and destabilization of vulnerable plaques in a rabbit model of atherosclerosis, Eur J Pharmacol 672(1-3) (2011) 143-52.

[9] G. Yang, Y. Li, W. Wu, B. Liu, L. Ni, Z. Wang, S. Miao, L. Wang, C. Liu, Anti-oxidant effect of heme oxygenase-1 on cigarette smoke-induced vascular injury, Mol Med Rep 12(2) (2015) 2481-6. 
[10] C.F. Chang, X.M. Liu, K.J. Peyton, W. Durante, Heme oxygenase-1 counteracts contrast media-induced endothelial cell dysfunction, Biochem Pharmacol 87(2) (2014) 303-11.

[11] N.G. Abraham, T. Kushida, J. McClung, M. Weiss, S. Quan, R. Lafaro, Z. Darzynkiewicz, M. Wolin, Heme oxygenase-1 attenuates glucose-mediated cell growth arrest and apoptosis in human microvessel endothelial cells, Circ Res 93(6) (2003) 507-14.

[12] A.L. Kruger, S.J. Peterson, M.L. Schwartzman, H. Fusco, J.A. McClung, M. Weiss, S. Shenouda, A.I. Goodman, M.S. Goligorsky, A. Kappas, N.G. Abraham, Up-regulation of heme oxygenase provides vascular protection in an animal model of diabetes through its antioxidant and antiapoptotic effects, J Pharmacol Exp Ther 319(3) (2006) 1144-52.

[13] U. Ozcan, Q. Cao, E. Yilmaz, A.H. Lee, N.N. Iwakoshi, E. Ozdelen, G. Tuncman, C. Gorgun, L.H. Glimcher, G.S. Hotamisligil, Endoplasmic reticulum stress links obesity, insulin action, and type 2 diabetes, Science 306(5695) (2004) 457-61.

[14] M. Kassan, M. Galan, M. Partyka, Z. Saifudeen, D. Henrion, M. Trebak, K. Matrougui, Endoplasmic reticulum stress is involved in cardiac damage and vascular endothelial dysfunction in hypertensive mice, Arterioscler Thromb Vasc Biol 32(7) (2012) 1652-61.

[15] M. Flamment, E. Hajduch, P. Ferre, F. Foufelle, New insights into ER stress-induced insulin resistance, Trends Endocrinol Metab 23(8) (2012) 381-90.

[16] R. Sano, J.C. Reed, ER stress-induced cell death mechanisms, Biochim Biophys Acta 1833(12) (2013) 3460-70.

[17] M. Redza-Dutordoir, D.A. Averill-Bates, Activation of apoptosis signalling pathways by reactive oxygen species, Biochim Biophys Acta (2016).

[18] A. Agouni, N. Mody, C. Owen, A. Czopek, D. Zimmer, M. Bentires-Alj, K.K. Bence, M. Delibegovic, Liver-specific deletion of protein tyrosine phosphatase (PTP) 1B improves obesity- and pharmacologically induced endoplasmic reticulum stress, Biochem J 438(2) (2011) 369-78. 
[19] T. Minamino, M. Kitakaze, ER stress in cardiovascular disease, J Mol Cell Cardiol 48(6) (2010) 1105-10.

[20] U. Ozcan, E. Yilmaz, L. Ozcan, M. Furuhashi, E. Vaillancourt, R.O. Smith, C.Z. Gorgun, G.S. Hotamisligil, Chemical chaperones reduce ER stress and restore glucose homeostasis in a mouse model of type 2 diabetes, Science 313(5790) (2006) 1137-40.

[21] M. Galan, M. Kassan, S.K. Choi, M. Partyka, M. Trebak, D. Henrion, K. Matrougui, A novel role for epidermal growth factor receptor tyrosine kinase and its downstream endoplasmic reticulum stress in cardiac damage and microvascular dysfunction in type 1 diabetes mellitus, Hypertension 60(1) (2012) 71-80.

[22] M.K. Sardana, A. Kappas, Dual control mechanism for heme oxygenase: tin(IV)protoporphyrin potently inhibits enzyme activity while markedly increasing content of enzyme protein in liver, Proc Natl Acad Sci U S A 84(8) (1987) 2464-8.

[23] A. Agouni, S. Tual-Chalot, M. Chalopin, L. Duluc, N. Mody, M.C. Martinez, R. Andriantsitohaina, M. Delibegovic, Hepatic protein tyrosine phosphatase 1B (PTP1B) deficiency protects against obesity-induced endothelial dysfunction, Biochem Pharmacol 92(4) (2014) 607-17.

[24] A. Alnabulsi, A. Agouni, S. Mitra, I. Garcia-Murillas, B. Carpenter, S. Bird, G.I. Murray, Cellular apoptosis susceptibility (chromosome segregation 1-like, CSE1L) gene is a key regulator of apoptosis, migration and invasion in colorectal cancer, J Pathol (2012).

[25] H.A. Mostefai, A. Agouni, N. Carusio, M.L. Mastronardi, C. Heymes, D. Henrion, R. Andriantsitohaina, M.C. Martinez, Phosphatidylinositol 3-kinase and xanthine oxidase regulate nitric oxide and reactive oxygen species productions by apoptotic lymphocyte microparticles in endothelial cells, J Immunol 180(7) (2008) 5028-35. 
[26] A. Agouni, H.A. Mostefai, C. Porro, N. Carusio, J. Favre, V. Richard, D. Henrion, M.C. Martinez, R. Andriantsitohaina, Sonic hedgehog carried by microparticles corrects endothelial injury through nitric oxide release, Faseb J 21(11) (2007) 2735-41.

[27] T. Benameur, S. Tual-Chalot, R. Andriantsitohaina, M.C. Martinez, PPARalpha is essential for microparticle-induced differentiation of mouse bone marrow-derived endothelial progenitor cells and angiogenesis, PLoS One 5(8) (2010) e12392.

[28] B.M. Babior, The NADPH oxidase of endothelial cells, IUBMB Life 50(4-5) (2000) 267-9.

[29] O. Perisic, M.I. Wilson, D. Karathanassis, J. Bravo, M.E. Pacold, C.D. Ellson, P.T. Hawkins, L. Stephens, R.L. Williams, The role of phosphoinositides and phosphorylation in regulation of NADPH oxidase, Adv Enzyme Regul 44 (2004) 279-98.

[30] N. Ferrara, Role of vascular endothelial growth factor in regulation of physiological angiogenesis, Am J Physiol Cell Physiol 280(6) (2001) C1358-66.

[31] B. Basha, S.M. Samuel, C.R. Triggle, H. Ding, Endothelial dysfunction in diabetes mellitus: possible involvement of endoplasmic reticulum stress?, Exp Diabetes Res 2012 (2012) 481840.

[32] A.D. Mooradian, M.J. Haas, Glucose-induced endoplasmic reticulum stress is independent of oxidative stress: A mechanistic explanation for the failure of antioxidant therapy in diabetes, Free Radic Biol Med 50(9) (2011) 1140-3.

[33] K.M. Kim, H.O. Pae, M. Zheng, R. Park, Y.M. Kim, H.T. Chung, Carbon monoxide induces heme oxygenase-1 via activation of protein kinase R-like endoplasmic reticulum kinase and inhibits endothelial cell apoptosis triggered by endoplasmic reticulum stress, Circ Res 101(9) (2007) 919-27.

[34] S. Lenna, R. Han, M. Trojanowska, Endoplasmic reticulum stress and endothelial dysfunction, IUBMB Life 66(8) (2014) 530-7. 
[35] K. Sakaki, R.J. Kaufman, Regulation of ER stress-induced macroautophagy by protein kinase C, Autophagy 4(6) (2008) 841-3.

[36] L. Teng, L.M. Fan, D. Meijles, J.M. Li, Divergent effects of p47(phox) phosphorylation at S303-4 or S379 on tumor necrosis factor-alpha signaling via TRAF4 and MAPK in endothelial cells, Arterioscler Thromb Vasc Biol 32(6) (2012) 1488-96.

[37] K. Bedard, K.H. Krause, The NOX family of ROS-generating NADPH oxidases: physiology and pathophysiology, Physiol Rev 87(1) (2007) 245-313.

[38] A.M. Schmidt, O. Hori, J.X. Chen, J.F. Li, J. Crandall, J. Zhang, R. Cao, S.D. Yan, J. Brett, D. Stern, Advanced glycation endproducts interacting with their endothelial receptor induce expression of vascular cell adhesion molecule-1 (VCAM-1) in cultured human endothelial cells and in mice. A potential mechanism for the accelerated vasculopathy of diabetes, J Clin Invest 96(3) (1995) 1395-403.

[39] K. Zhang, X. Shen, J. Wu, K. Sakaki, T. Saunders, D.T. Rutkowski, S.H. Back, R.J. Kaufman, Endoplasmic reticulum stress activates cleavage of CREBH to induce a systemic inflammatory response, Cell 124(3) (2006) 587-99.

[40] I. Tabas, D. Ron, Integrating the mechanisms of apoptosis induced by endoplasmic reticulum stress, Nat Cell Biol 13(3) (2011) 184-90.

[41] E. Iori, E. Pagnin, A. Gallo, L. Calo, E. Murphy, F. Ostuni, G.P. Fadini, A. Avogaro, Heme oxygenase-1 is an important modulator in limiting glucose-induced apoptosis in human umbilical vein endothelial cells, Life Sci 82(7-8) (2008) 383-92.

[42] H. Urra, E. Dufey, F. Lisbona, D. Rojas-Rivera, C. Hetz, When ER stress reaches a dead end, Biochim Biophys Acta 1833(12) (2013) 3507-17.

[43] A.H. Chaanine, D. Jeong, L. Liang, E.R. Chemaly, K. Fish, R.E. Gordon, R.J. Hajjar, JNK modulates FOXO3a for the expression of the mitochondrial death and mitophagy marker BNIP3 in pathological hypertrophy and in heart failure, Cell Death Dis 3 (2012) 265. 
[44] G. Li Volti, D. Sacerdoti, B. Sangras, A. Vanella, A. Mezentsev, G. Scapagnini, J.R. Falck, N.G. Abraham, Carbon monoxide signaling in promoting angiogenesis in human microvessel endothelial cells, Antioxid Redox Signal 7(5-6) (2005) 704-10.

[45] A. Amin, S.K. Choi, M. Galan, M. Kassan, M. Partyka, P. Kadowitz, D. Henrion, M. Trebak, S. Belmadani, K. Matrougui, Chronic inhibition of endoplasmic reticulum stress and inflammation prevents ischaemia-induced vascular pathology in type II diabetic mice, $\mathrm{J}$ Pathol 227(2) (2012) 165-74.

[46] D. Fukumura, T. Gohongi, A. Kadambi, Y. Izumi, J. Ang, C.O. Yun, D.G. Buerk, P.L. Huang, R.K. Jain, Predominant role of endothelial nitric oxide synthase in vascular endothelial growth factor-induced angiogenesis and vascular permeability, Proc Natl Acad Sci U S A 98(5) (2001) 2604-9.

[47] C. Loinard, Y. Zouggari, P. Rueda, B. Ramkhelawon, C. Cochain, J. Vilar, A. Recalde, A. Richart, D. Charue, M. Duriez, M. Mori, F. Arenzana-Seisdedos, B.I. Levy, C. Heymes, J.S. Silvestre, C/EBP homologous protein-10 (CHOP-10) limits postnatal neovascularization through control of endothelial nitric oxide synthase gene expression, Circulation 125(8) (2012) 1014-26.

[48] G. Zeng, F.H. Nystrom, L.V. Ravichandran, L.N. Cong, M. Kirby, H. Mostowski, M.J. Quon, Roles for insulin receptor, PI3-kinase, and Akt in insulin-signaling pathways related to production of nitric oxide in human vascular endothelial cells, Circulation 101(13) (2000) $1539-45$.

[49] E.R. Duncan, P.A. Crossey, S. Walker, N. Anilkumar, L. Poston, G. Douglas, V.A. Ezzat, S.B. Wheatcroft, A.M. Shah, M.T. Kearney, Effect of endothelium-specific insulin resistance on endothelial function in vivo, Diabetes 57(12) (2008) 3307-14.

[50] N.G. Abraham, J.M. Junge, G.S. Drummond, Translational Significance of Heme Oxygenase in Obesity and Metabolic Syndrome, Trends Pharmacol Sci 37(1) (2016) 17-36. 
[51] H. Dong, H. Huang, X. Yun, D.S. Kim, Y. Yue, H. Wu, A. Sutter, K.D. Chavin, L.E. Otterbein, D.B. Adams, Y.B. Kim, H. Wang, Bilirubin increases insulin sensitivity in leptinreceptor deficient and diet-induced obese mice through suppression of ER stress and chronic inflammation, Endocrinology 155(3) (2014) 818-28.

[52] J.E. Clark, R. Foresti, P. Sarathchandra, H. Kaur, C.J. Green, R. Motterlini, Heme oxygenase-1-derived bilirubin ameliorates postischemic myocardial dysfunction, Am J Physiol Heart Circ Physiol 278(2) (2000) H643-51.

[53] M. Zheng, Q. Zhang, Y. Joe, S.K. Kim, M.J. Uddin, H. Rhew, T. Kim, S.W. Ryter, H.T. Chung, Carbon monoxide-releasing molecules reverse leptin resistance induced by endoplasmic reticulum stress, Am J Physiol Endocrinol Metab 304(7) (2013) E780-8. 


\section{Figure Legends:}

Figure 1. Validation of conditions of HO-1 induction by CoPP. (A), western blot analysis of HO-1 and $\beta$-actin expression in HUVECs treated with CoPP $(10,25,50,75$ and $100 \mu \mathrm{M})$ for 12 or 24 hours. Representative image from 3 independent experiments. (B), viability (MTT) assay of HUVECs treated with $\operatorname{CoPP}(10,25,50,75$ and $100 \mu \mathrm{M})$ for 12 or 24 hours. Histograms represent pooled normalized data to negative control (CTL) ( $n=3-6$ per group). (C), HO-1 activity assay showing bilirubin production $(\mathrm{mg} / \mathrm{dL})$ in HUVECs incubated in physiological $(5 \mathrm{mM})$ concentration of glucose or in intermittent high glucose $(5 / 33 \mathrm{mM})$ for 5 days in the presence or absence of $\mathrm{CoPP}$ or $\operatorname{SnPP}(n=3)$. Data are presented as mean $\pm \operatorname{SEM}$ and were analysed by one-way ANOVA, followed with a Tukey's multiple comparison test. ${ }^{*} P<0.05,{ }^{* *} P<0.01,{ }^{* * *} P<0.001$ vs. control or indicated groups. CTL, control; HG, high glucose.

Figure 2. HO-1 induction in HUVECs attenuates high glucose-induced ER stress. (A, B), Relative mRNA expression levels of ER stress target markers BiP (A), CHOP (B) and ATF-4 (C) normalised against $\beta$-actin housekeeping gene $(n=6)$. (C, D), western blot analysis showing protein expression levels of BiP, p-eIF2 $\alpha$ (D and E) and ATF-4 (F). Histograms represent pooled normalized data to total amount of normalised to $\beta$-actin loading control expressed as arbitrary units (A.U.) ( $\mathrm{n}=3$ per group). Data are presented as mean \pm SEM and were analysed by one-way ANOVA, followed with a Tukey's multiple comparison test. ${ }^{*} P<0.05,{ }^{* *} P<0.01,{ }^{* * *} P<0.001$ vs. control or indicated groups. CTL, control; HG, high glucose; TG, thapsigargin; Man, Mannitol.

Figure 3. HO-1 induction in HUVECs protects against high glucose-induced oxidative stress. (A), Measurement of ROS production in HUVECs. Cells were either treated with $\mathrm{H}_{2} \mathrm{O}_{2}(100 \mu \mathrm{M})$ for 30 minutes (PC) or incubated with intermittent high glucose (HG) for 5 days in the presence or absence of PBA, CoPP or Mannitol. In negative control (NC) group, 
cells where incubated with M200 medium only. Histograms represent the percentage of DHE-positive cells to negative control. (B), western blot analysis of protein expression of phosphorylated $\mathrm{p} 47^{\text {phox }}$ (Ser345). Histograms represent pooled normalized data to total amount of total protein $\mathrm{p} 47^{\text {phox }}$ expressed as arbitrary units (A.U.) (n=4). Data are presented as mean \pm SEM and were analysed by one-way ANOVA, followed with a Tukey's multiple comparison test. ${ }^{* *} P<0.01,{ }^{* * *} P<0.001$ vs. Control or indicated groups. CTL, control; HG, high glucose; PC, positive control; NC, negative control; TG, thapsigargin; Man, Mannitol.

Figure 4. HO-1 induction in HUVECs prevents inflammatory response mediated by high glucose-induced ER stress. (A, B), western blot analysis showing protein expression levels of $\mathrm{p}$-iKK $\alpha / \beta$ (A) normalized against iKK and $\mathrm{p}$-c-JUN normalized against $\beta$-actin (B). Histograms represent pooled normalized data to total amount of respective loading control expressed as arbitrary units (A.U.) ( $\mathrm{n}=4$ in each group). (C) Relative mRNA expression IL-6 normalized against $\beta$-actin housekeeping gene $(n=6)$. (D) ELISA analysis of IL-6 release in culture medium from HUVECs treated with LPS (PC) or high glucose (HG) in the presence or absence of CoPP and/or SnPP $(n=3)$. Data are presented as mean \pm SEM and were analyzed by one-way ANOVA, followed with a Tukey's multiple comparison test. ${ }^{*} P<0.05$, ${ }^{* *} P<0.01,{ }^{* * *} P<0.001$ vs. control or indicated groups. CTL, control; HG, high glucose; TG, thapsigargin; PC, positive control; Man, Mannitol.

Figure 5. HO-1 induction in HUVECs protects against high glucose-induced apoptosis. (A), Assessment of apoptosis by flow cytometry in HUVECs treated with intermittent high glucose (33 mM) using Annexin V and PI staining $(\mathrm{n}=3)$. Histograms represent pooled data and presented as percentage of control. Data are presented as mean \pm SEM and were analysed by one-way ANOVA, followed with a Tukey's multiple comparison. ${ }^{* * *} P<0.001$ vs. control or indicated groups. CTL, control; NT, negative treatment. 
Figure 6. HO-1 induction in HUVECs protects against high glucose-induced caspases 3/7 and PAPRP activation. (A), Caspase 3/7 Glo luminescent assay for assessment of Caspases 3 and 7 activity $(n=3)$. Cells treated with Staurosporine were used as positive control (PC). Untreated cells were used as negative control (NC). (B), western blot analysis to determine presence of cleaved PARP, caspase 3 and caspase 7. Images are representative of 4 independetnt experiments. Data are presented as mean \pm SEM and were analysed by oneway ANOVA, followed with a Tukey's multiple comparison. ${ }^{* * *} P<0.001$ vs. control or indicated groups. CTL, control; HG, high glucose; PC, positive control; NC, negative control; TG, thapsigargin; Man, Mannitol.

Figure 7. HO-1 induction improves NO bioavailability in in HUVECs incubated in high glucose. (A), Quantification of nitrite and nitrate productions in HUVECs. Cells were incubated in intermittent high glucose (HG) in the presence or absence of CoPP. Supernatants were collected and assayed for nitrite and nitrate production $(n=4)$. (B-C), western blot analysis of protein expression of p-eNOS (Ser1177) (B), p-Akt (Ser473) (C) and HO-1 (D). Histograms represent pooled normalized data to total amount of respective total protein expressed as arbitrary units (A.U.) ( $\mathrm{n}=4$ in each group). Data are presented as mean $\pm \mathrm{SEM}$ and were analysed by one-way ANOVA, followed with a Tukey's multiple comparison test. ${ }^{*} \mathrm{P}<0.05,{ }^{* *} \mathrm{P}<0.01,{ }^{* * *} \mathrm{P}<0.001$ vs. control or indicated groups. CTL, control; HG, high glucose.

Figure 8. Alleviation of high glucose-induced ER stress by HO-1 induction improves endothelial function and angiogenic capacity in HUVECs. (A), Phase-contrast micrographs of HUVECs incubated in complete culture M200 medium (positive control, PC), in basal culture medium negative control, NC) or in intermittent high glucose (HG) in the presence or absence of CoPP or PBA. Cells were grown on a Matrigel ${ }^{\circledR}$ matrix to favour network formation. Images are representative of 3 independent experiments (images taken 
after $24 \mathrm{~h}$ ). Capillary length was used to quantify angiogenesis in 5 different blind fields (Image $J$ software). Histograms represent pooled data and presented as average length of tubes formed in mm. (B), Relative mRNA expression of VEGF-A against GAPDH housekeeping gene $(\mathrm{n}=4)$. (C), western blot analysis of VEGF-A protein expression. Histograms represent pooled normalized data to total amount of $\beta$-actin expressed as arbitrary units (A.U.) ( $\mathrm{n}=4$ in each group). Data are presented as mean $\pm \mathrm{SEM}$ and were analysed by one-way ANOVA, followed with a Tukey's multiple comparison test. ${ }^{*} \mathrm{P}<0.05,{ }^{* *} \mathrm{P}<0.01$, ${ }^{* * *} \mathrm{P}<0.001$ vs. control or indicated groups. CTL, control; HG, high glucose; PC, positive control; NC, negative control. 
A

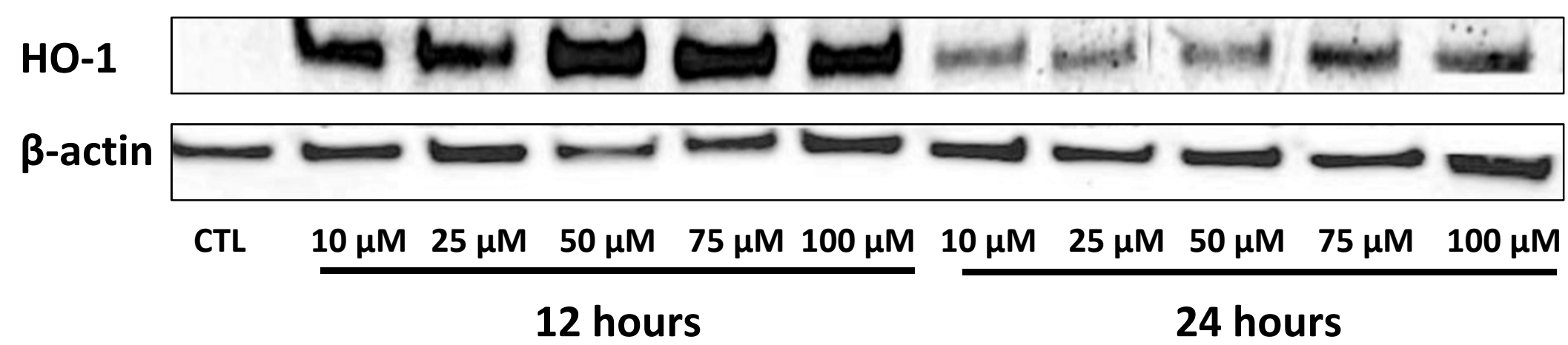

B

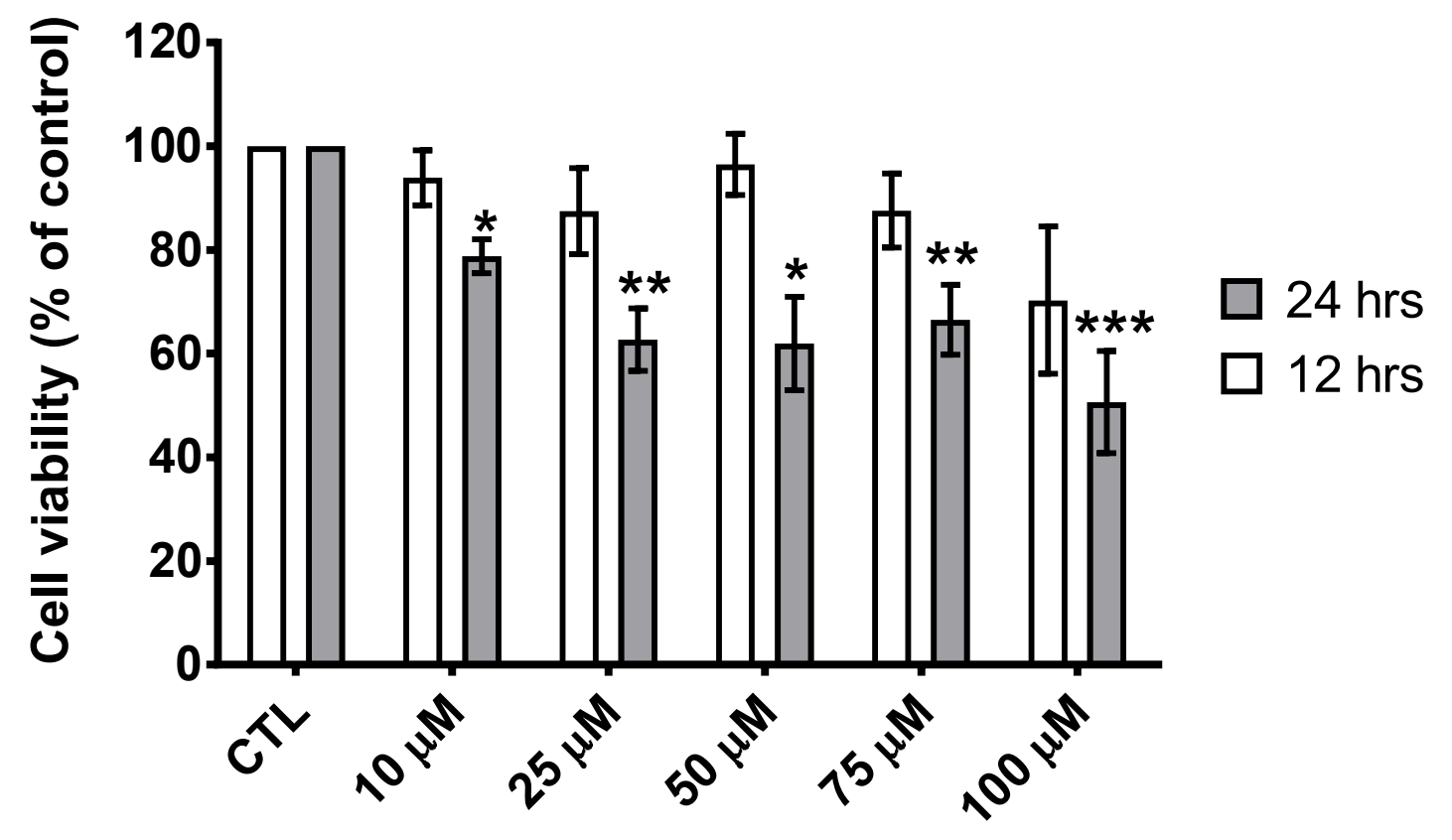

C

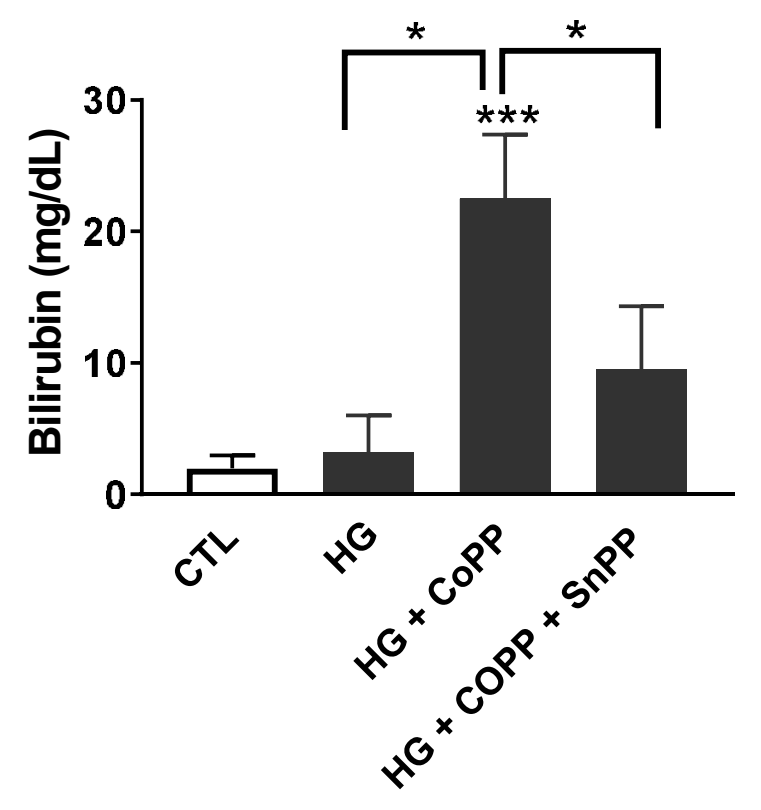

Figure 1 
A

B iP

B

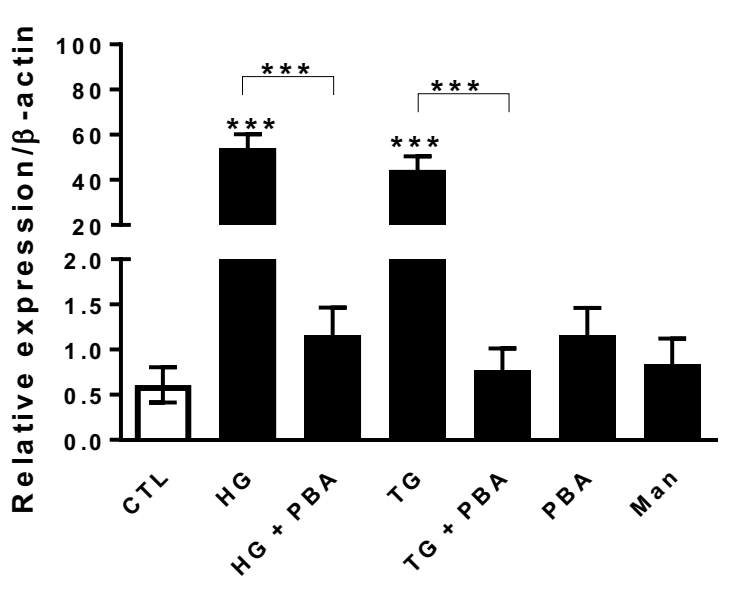

D

B iP

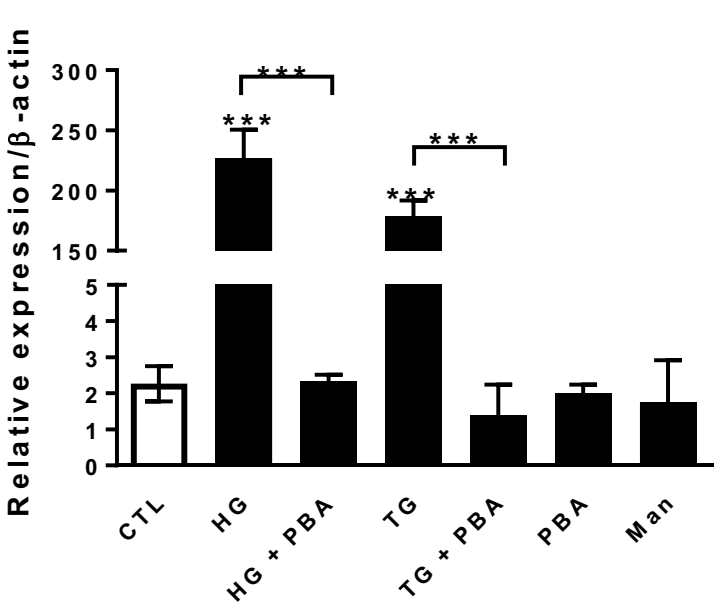

$E$
A TF -4

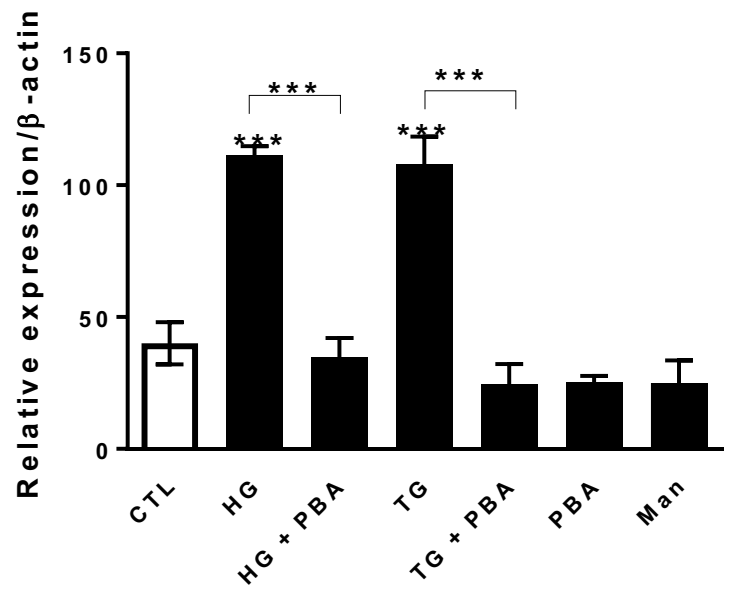

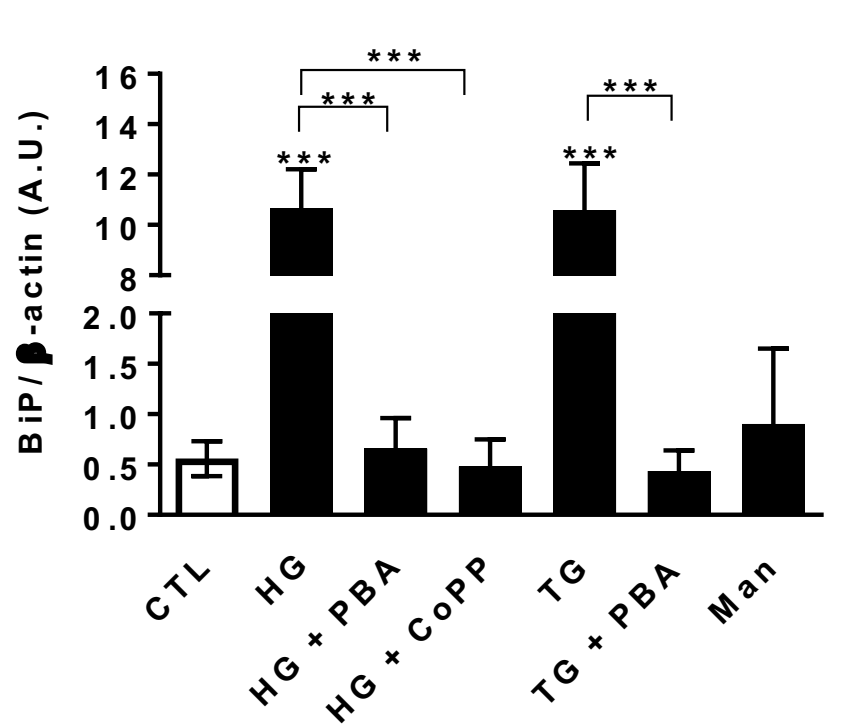

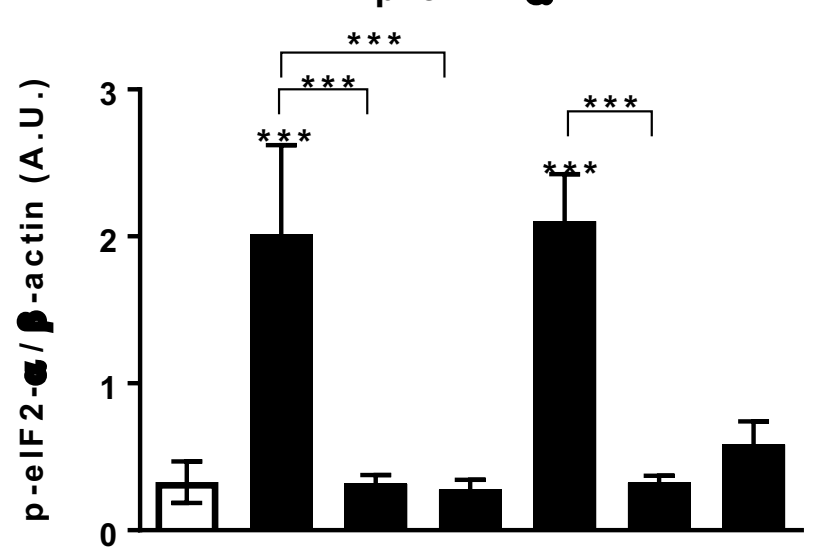

BiP

p-elF 2- $\alpha$

$\beta$-actin

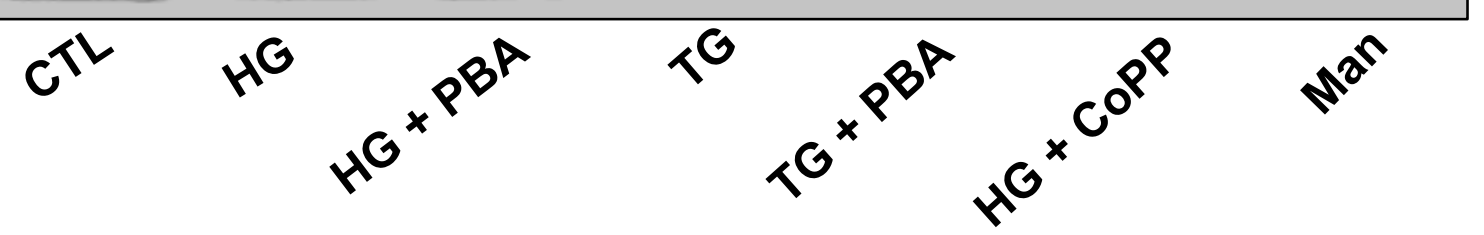

F

A T F -4

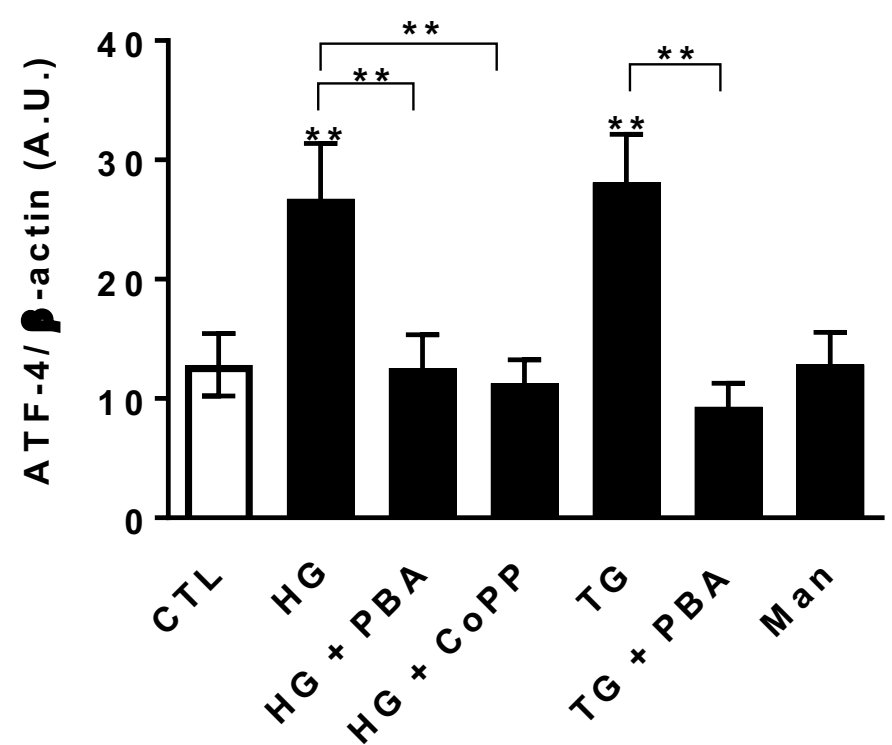

ATF-4

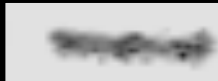

$\beta$-actin

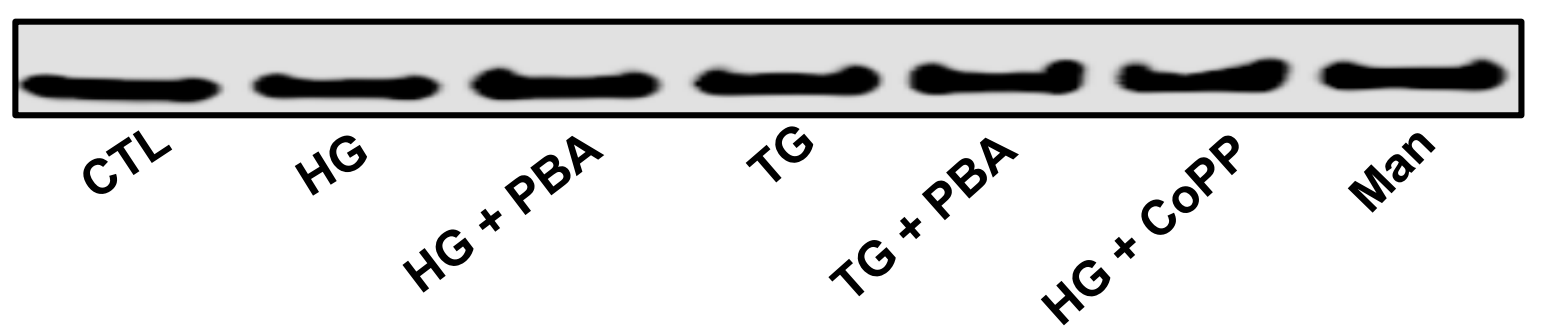

Figure 2 

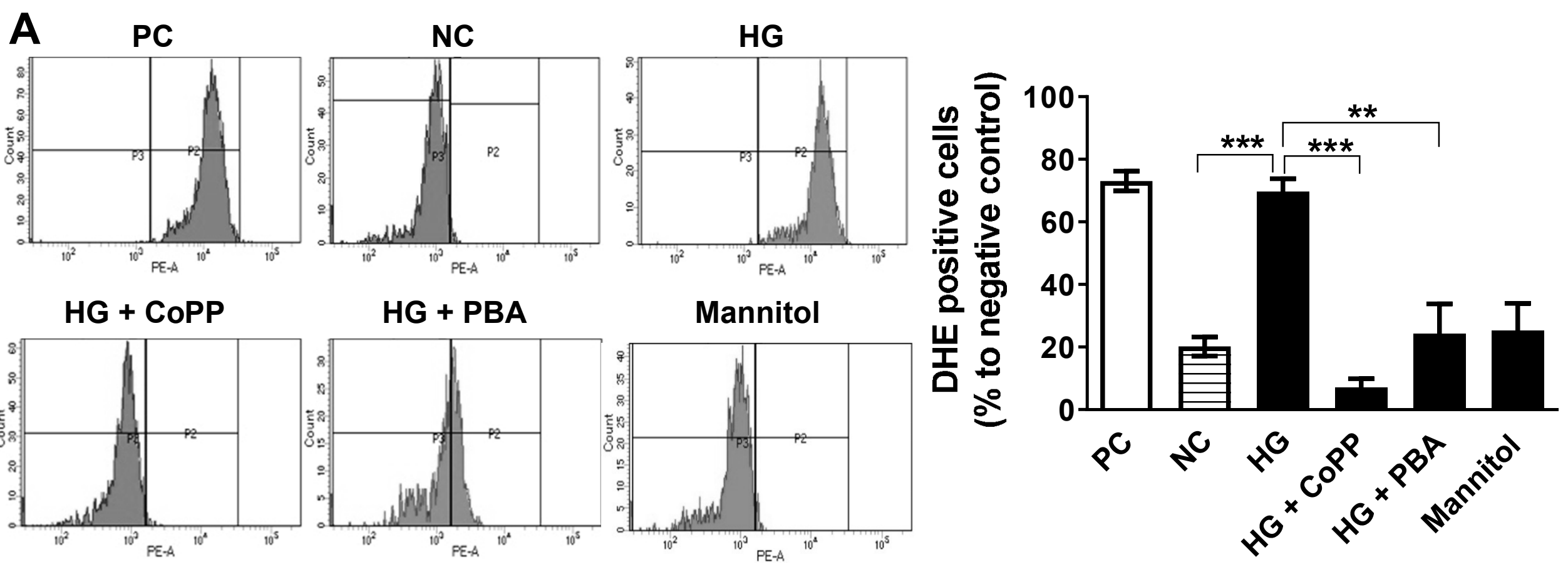

B

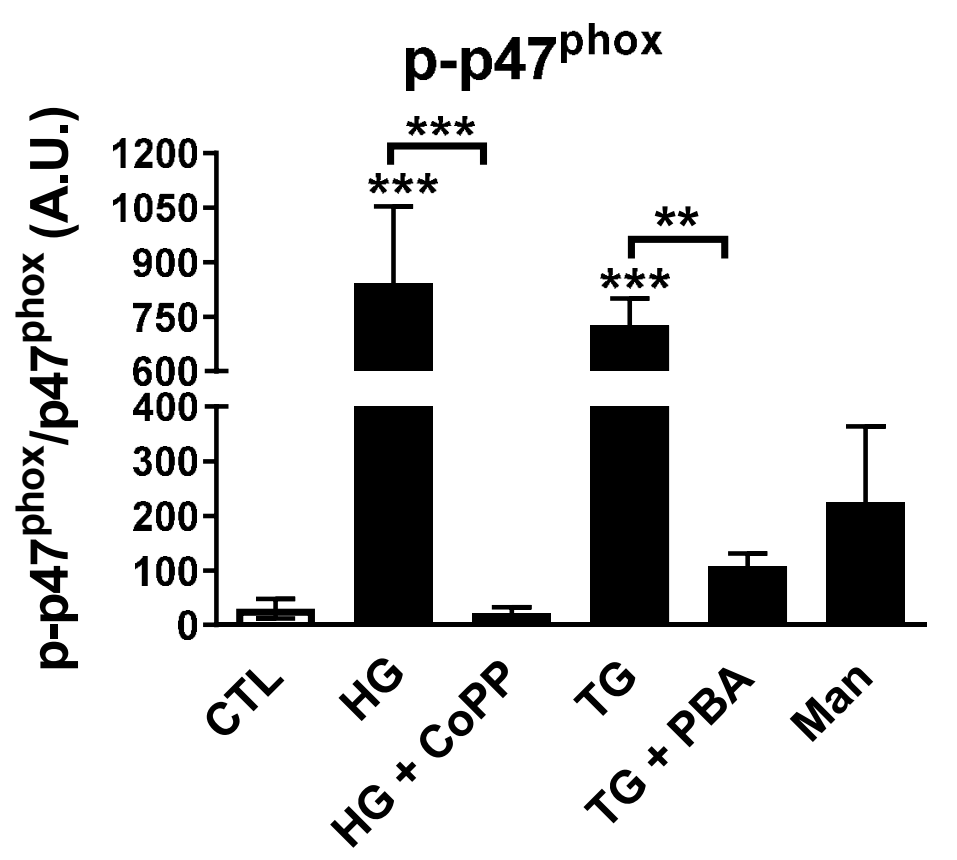

Figure 3 


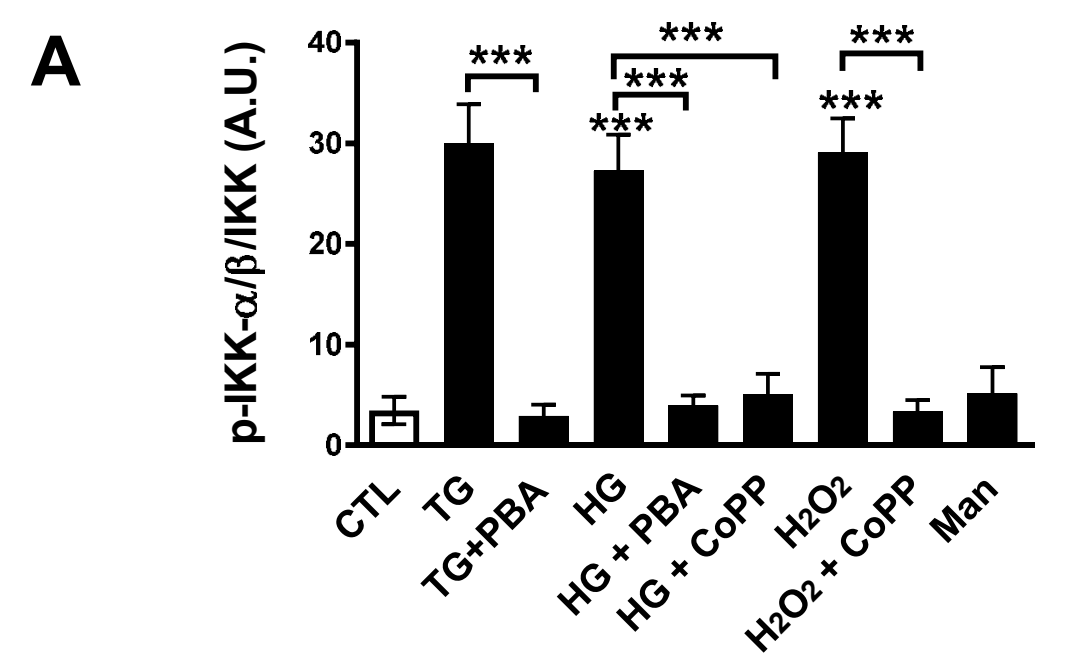

p-iKK $\alpha / \beta$

iKK
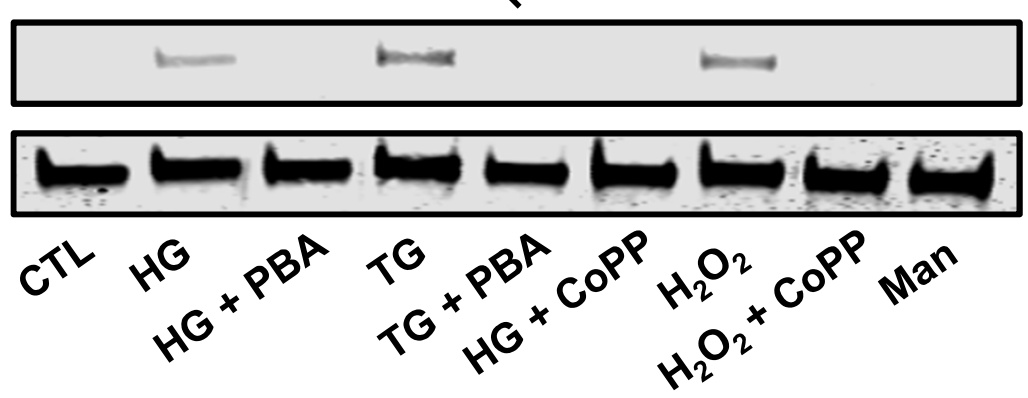

C

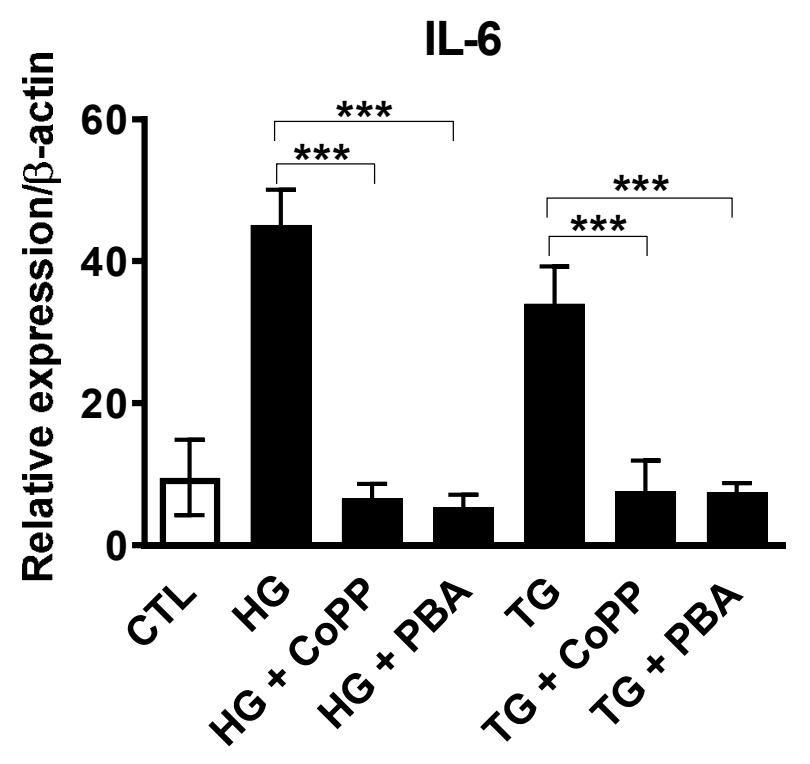

B

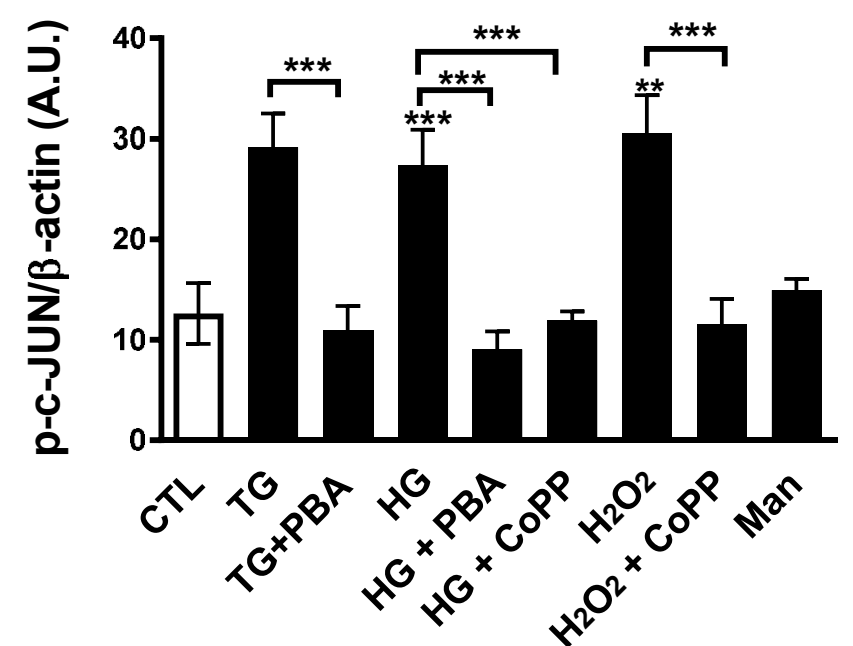

p-c-JUN

$\beta$-actin

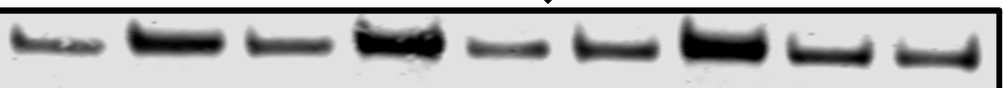

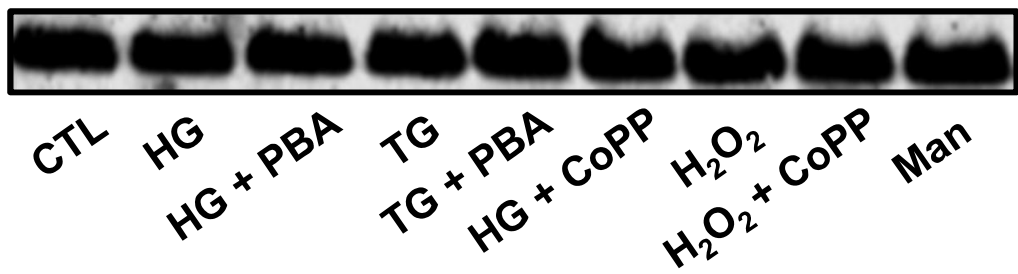

D

ELISA IL-6

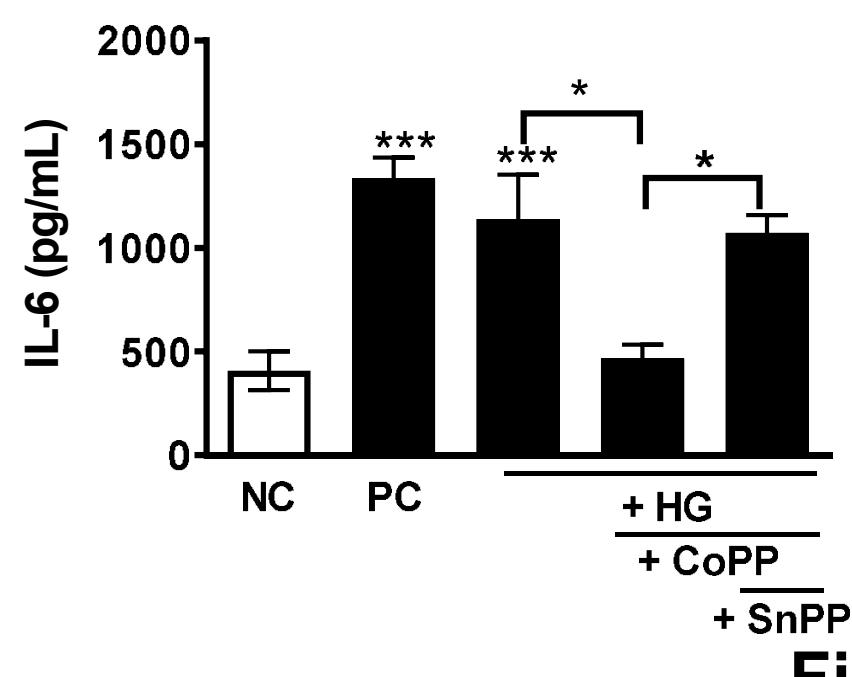

Figure 4 


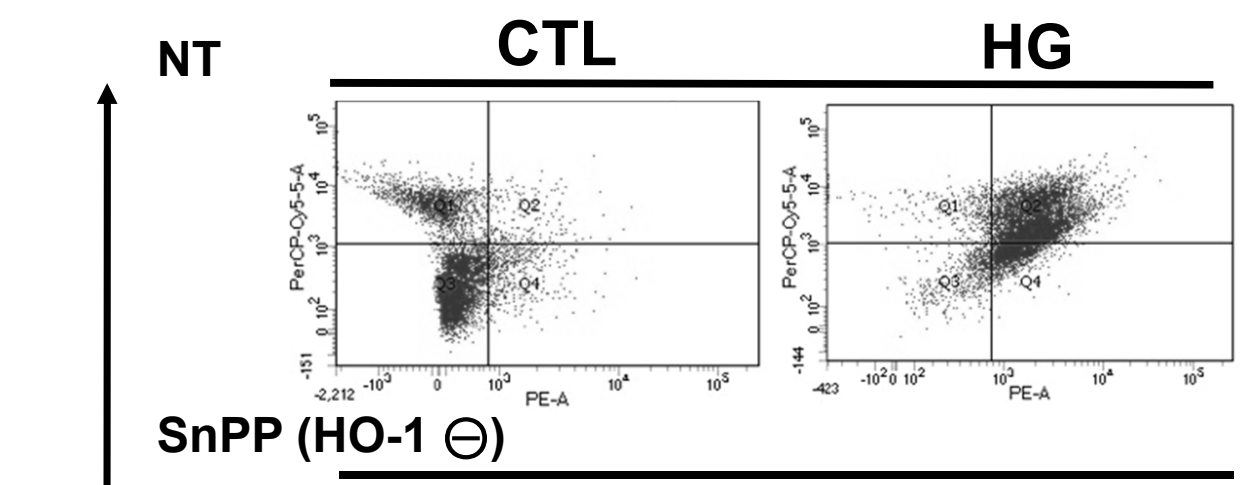

PI $\operatorname{CoPP}(\mathrm{HO}-1 \oplus)$
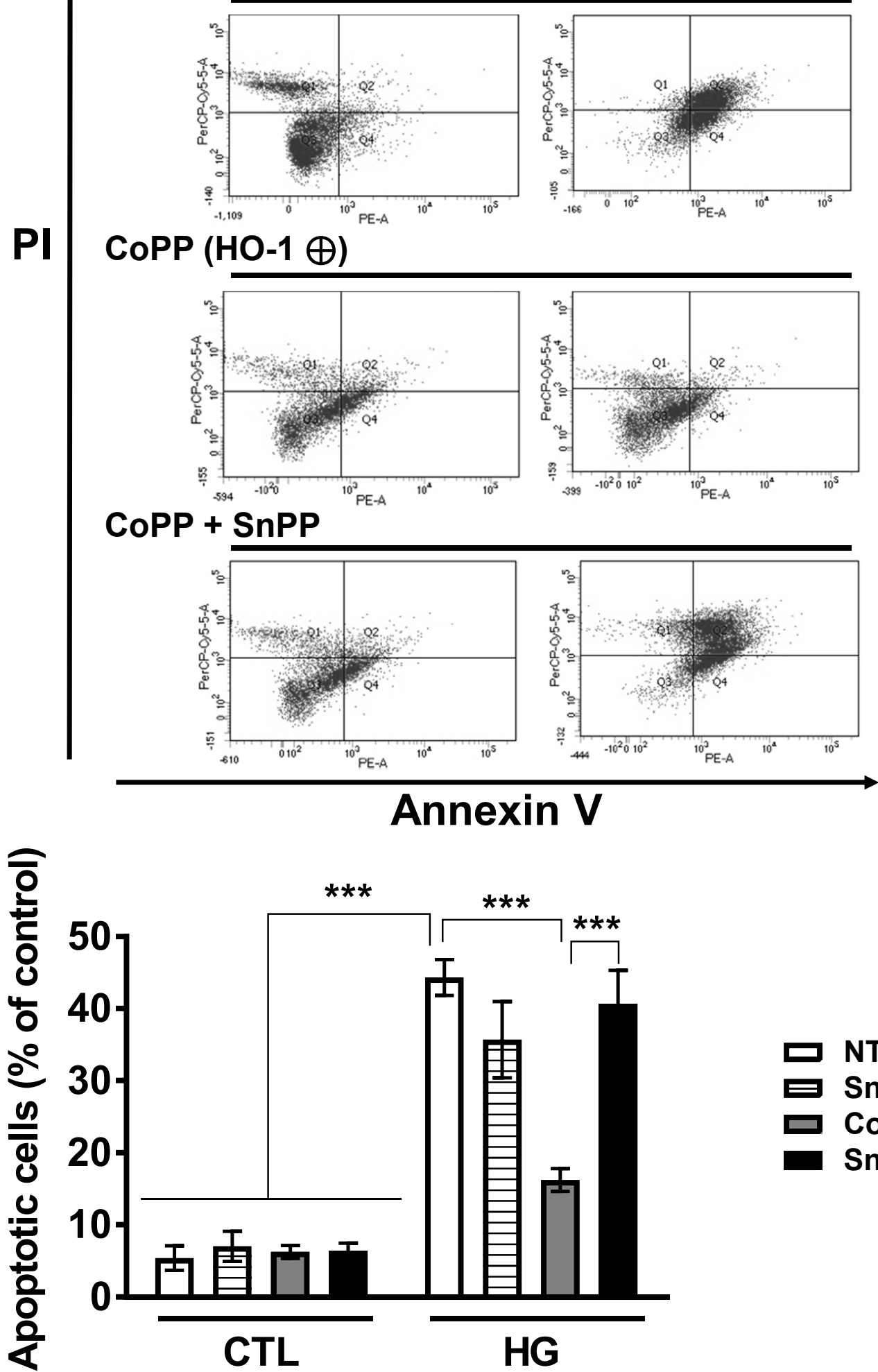

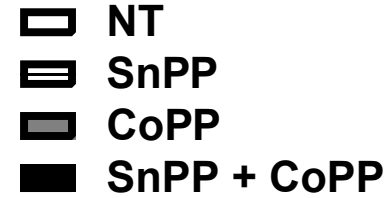

Figure 5 
A

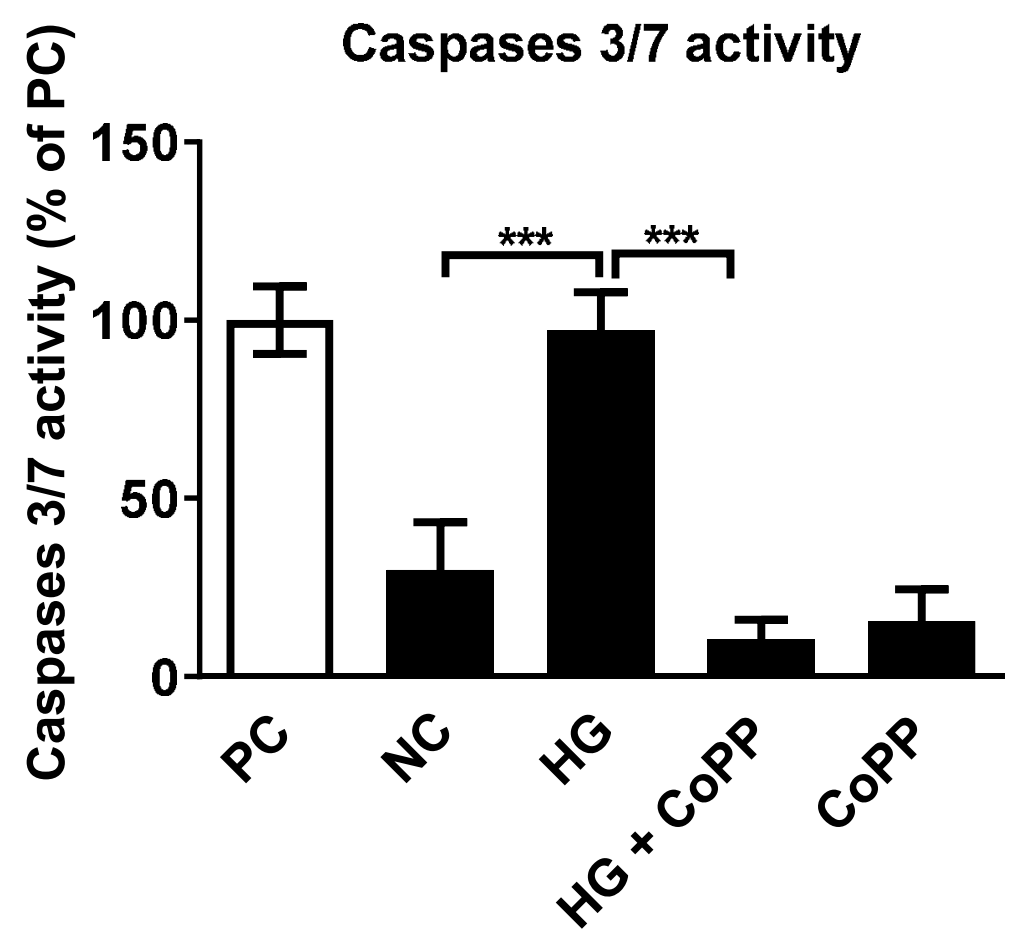

B

Caspases $3 / 7$ and PARP cleavage

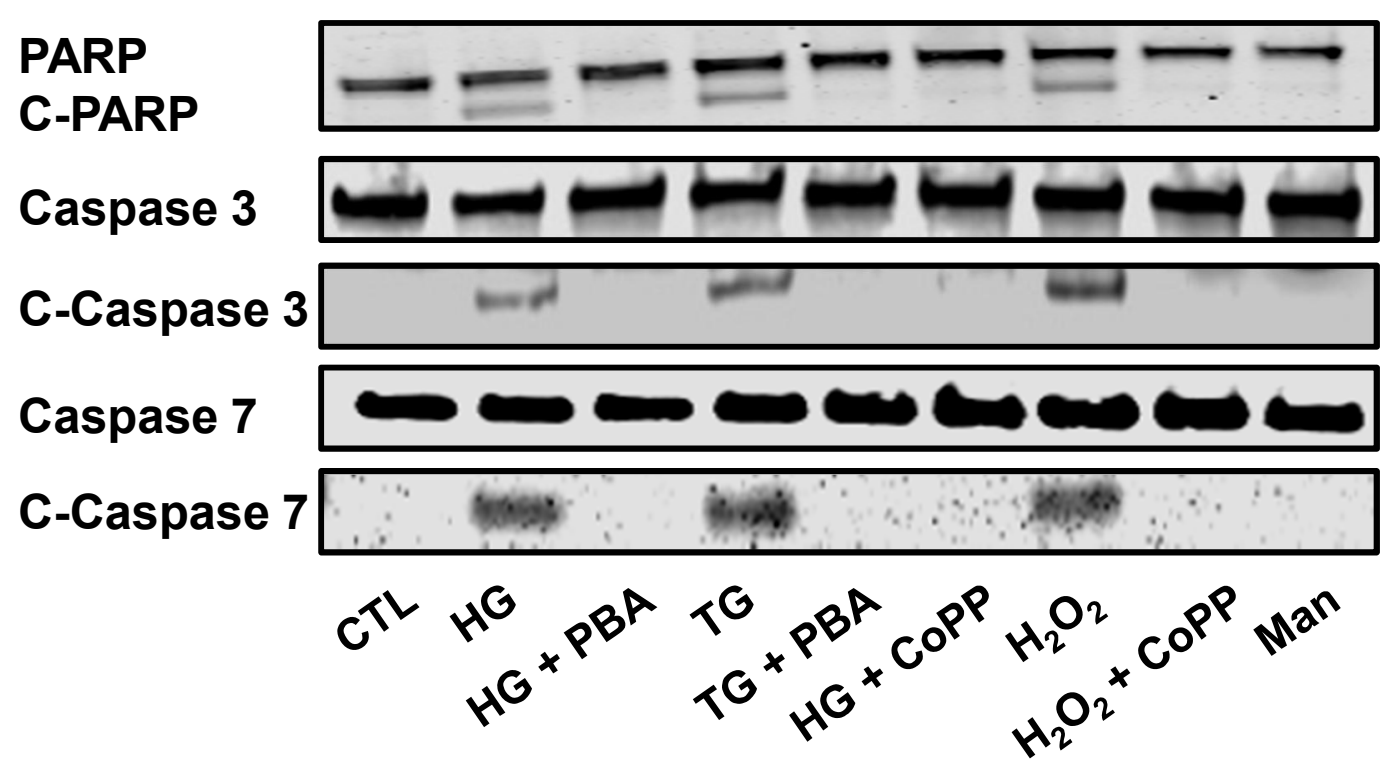

Figure 6 


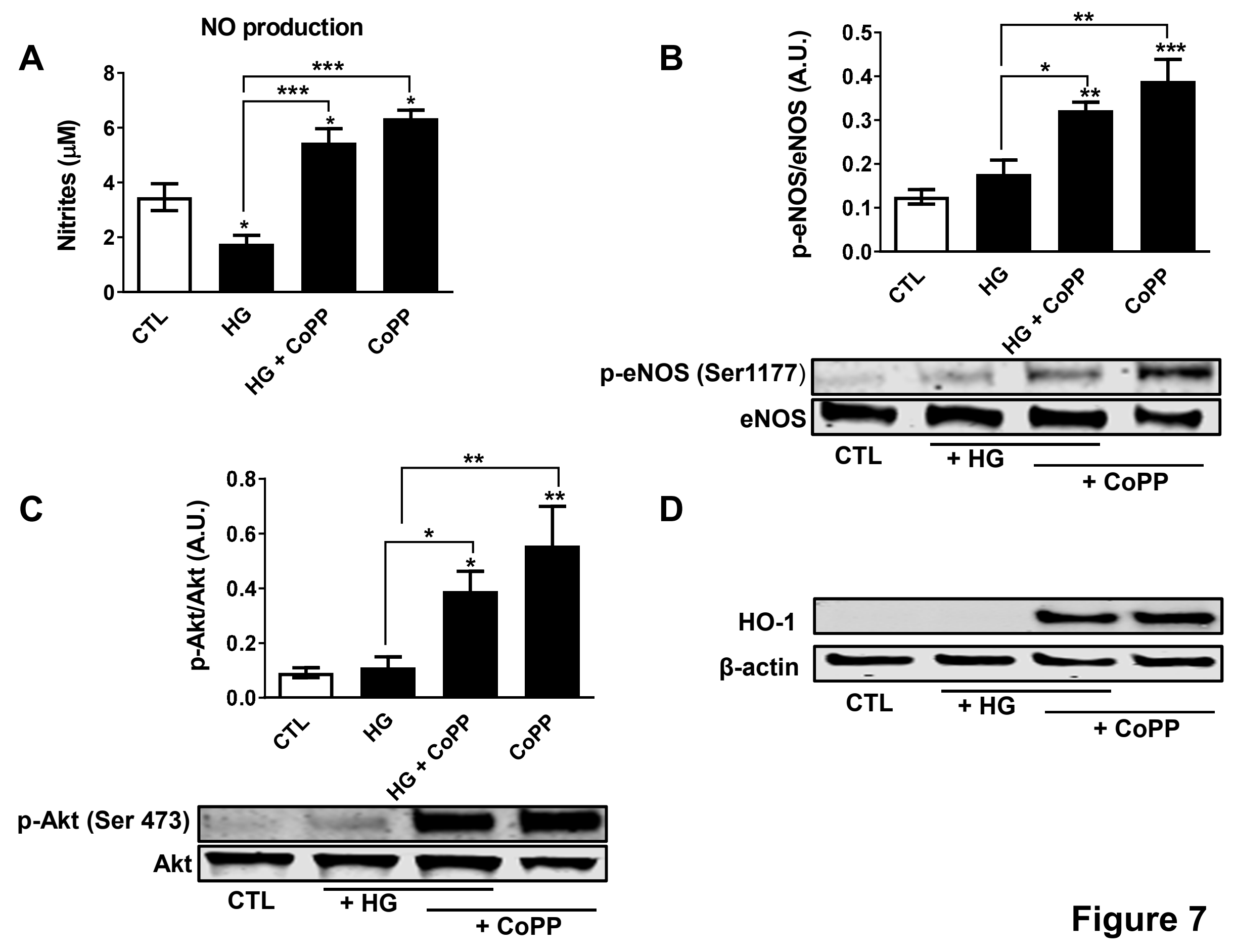


A

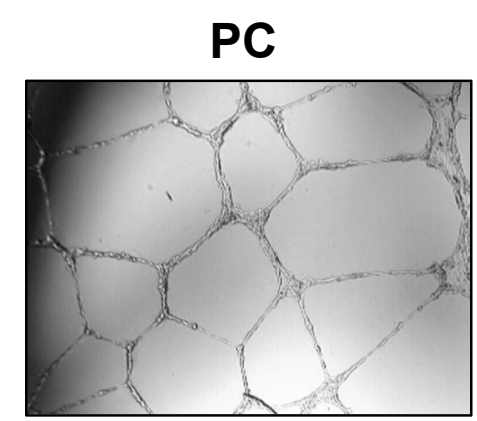

HG

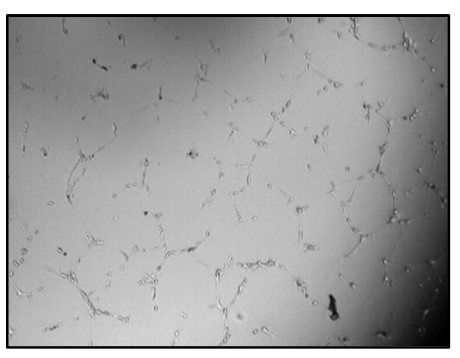

B

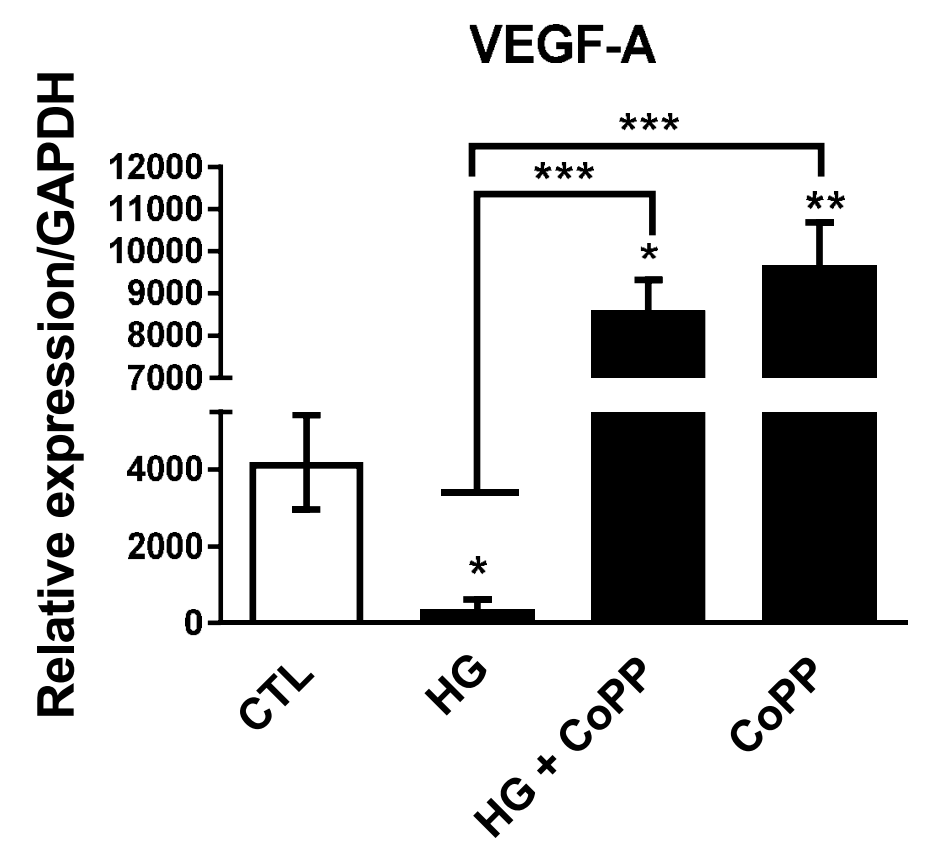

NC

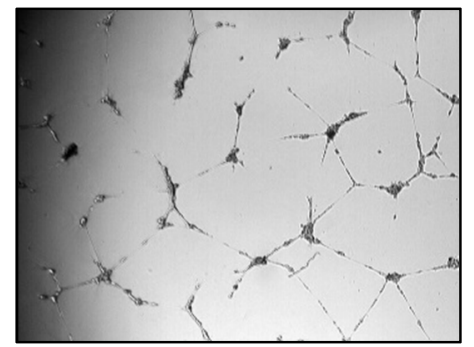

$$
\text { HG + CoPP }
$$

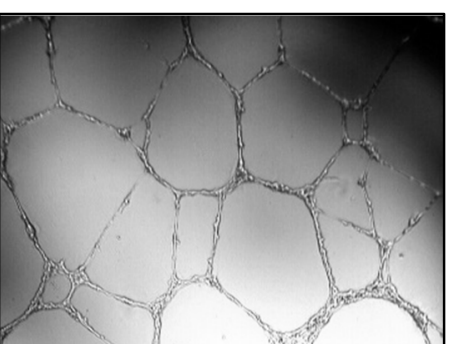

HG + PBA

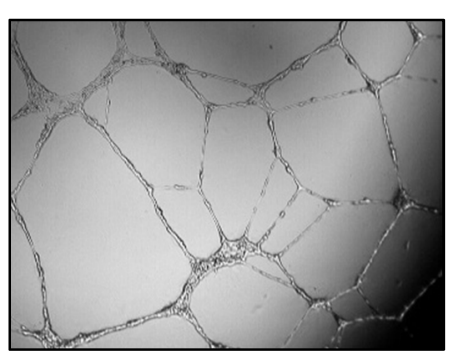

C
Tube formation assay
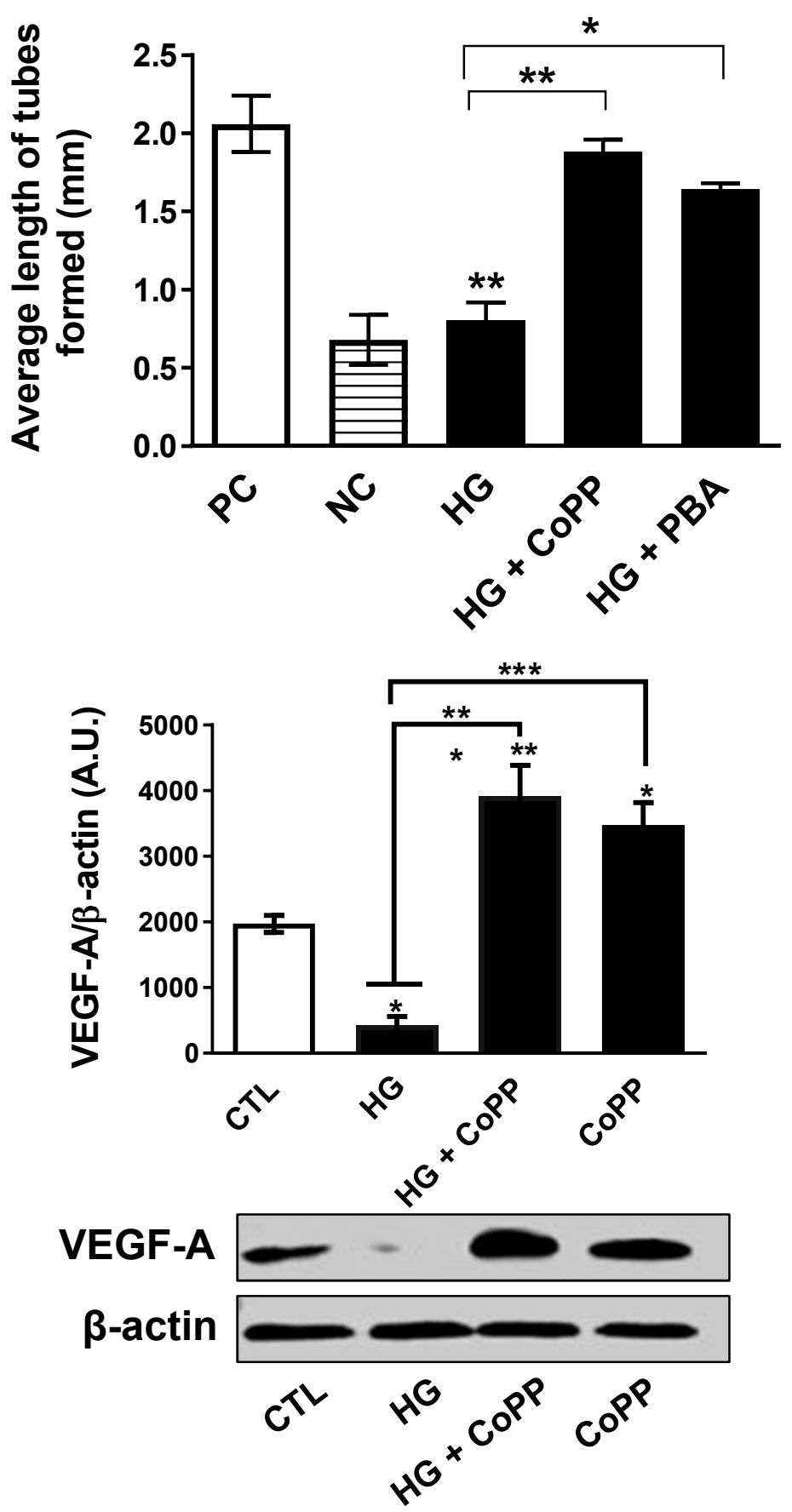

Figure 8 


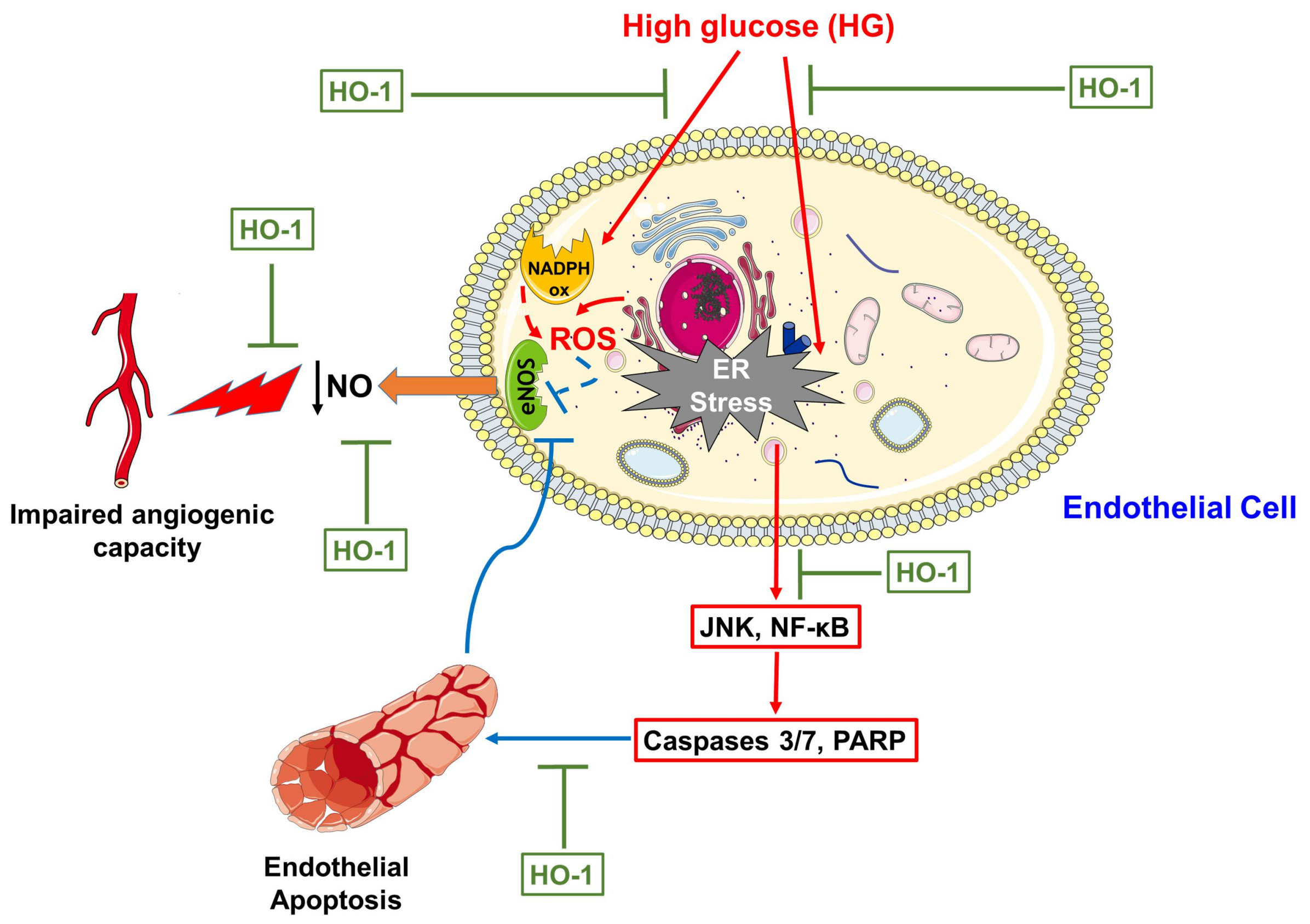

